\title{
3D CFD Model Validation Using Benchmark Data of 1/16 th Scaled VHTR Upper Plenum and Development of Wall Heat-Transfer Correlation For Laminar Flow
}

Nuclear Science and Engineering Division 


\begin{abstract}
About Argonne National Laboratory
Argonne is a U.S. Department of Energy laboratory managed by UChicago Argonne, LLC under contract DE-AC02-06CH11357. The Laboratory's main facility is outside Chicago, at 9700 South Cass Avenue, Argonne, Illinois 60439. For information about Argonne and its pioneering science and technology programs, see www.anl.gov.
\end{abstract}

\title{
DOCUMENT AVAILABILITY
}

Online Access: U.S. Department of Energy (DOE) reports produced after 1991 and a growing number of pre-1991 documents are available free via DOE’s SciTech Connect (http://www.osti.gov/scitech/)

Reports not in digital format may be purchased by the public from the

National Technical Information Service (NTIS):

U.S. Department of Commerce

National Technical Information Service

5301 Shawnee Rd Alexandra, VA 22312 www.ntis.gov

Phone: (800) 553-NTIS (6847) or (703) 605-6000

Fax: (703) 605-6900

Email: orders@ntis.gov

Reports not in digital format are available to DOE and DOE contractors from the Office of Scientific and Technical Information (OSTI):

U.S. Department of Energy

Office of Scientific and Technical Information

P.O. Box 62

Oak Ridge, TN 37831-0062

www.osti.gov

Phone: (865) 576-8401

Fax: (865) 576-5728

\footnotetext{
Disclaimer

This report was prepared as an account of work sponsored by an agency of the United States Government. Neither the United States Government nor any agency thereof, nor UChicago Argonne, LLC, nor any of their employees or officers, makes any warranty, express or implied, or assumes any legal liability or responsibility for the accuracy, completeness, or usefulness of any information, apparatus, product, or process disclosed, or represents that its use would not infringe privately owned rights. Reference herein to any specific commercial product, process, or service by trade name, trademark, manufacturer, or otherwise, does not necessarily constitute or imply its endorsement, recommendation, or favoring by the United States Government or any agency thereof. The views and opinions of document authors expressed herein do not necessarily state or reflect those of the United States Government or any agency thereof, Argonne National Laboratory, or UChicago Argonne, LLC.
} 


\section{D CFD Model Validation Using Benchmark Data of $1 / 16^{\text {th }}$ Scaled VHTR Upper Plenum and Development of Wall Heat-Transfer Correlation For Laminar Flow}

prepared by

Prasad Vegendla, Liu Yang, Ling Zou, Rui Hu

Nuclear Science and Engineering Division, Argonne National Laboratory

March 2020 
This page is intentionally left blank. 


\section{CONTENTS}

ACKNOWLEDGMENTS ......................................................................................... vi

ACRONYMS AND ABBREVIATIONS ......................................................................... vii

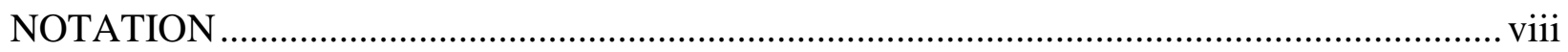

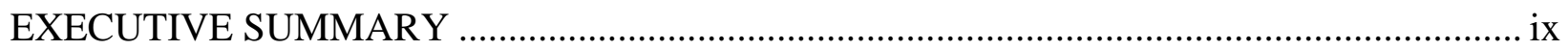

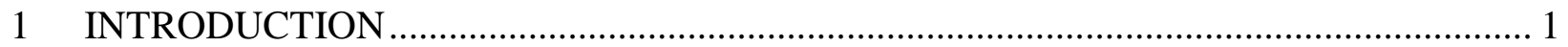

2 CFD MODEL VALIDATION FOR A BENCHMARK DATA OF TEXAS A\&M UNIVERSITY $1 / 16^{\text {th }}$ SCALED VHTR UPPER PLENUM.............................................. 4

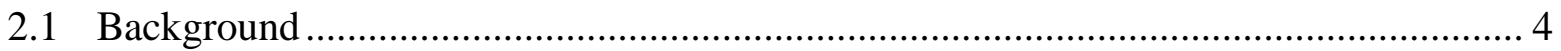

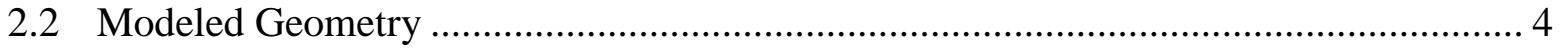

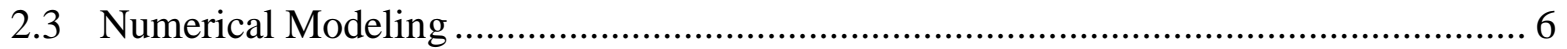

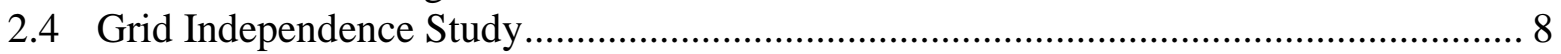

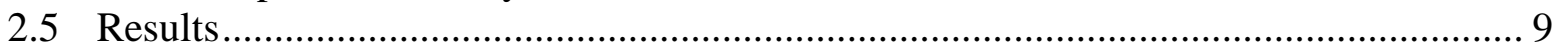

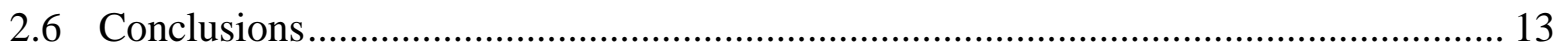

3 DEVELOPMENT OF HEAT TRANSFER CORRELATIONS FOR LAMINAR FLOW IN A WALL-HEATED CYLINDRICAL TUBE …....................................... 15

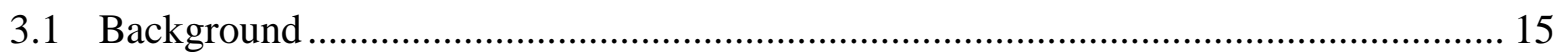

3.2 Matlab Regression Model .................................................................................. 17

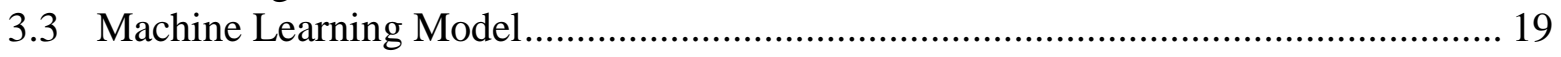

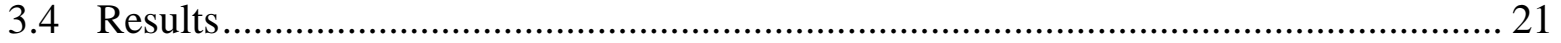

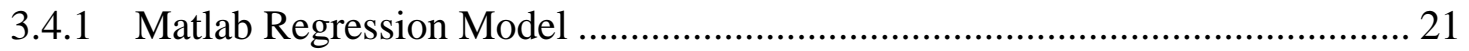

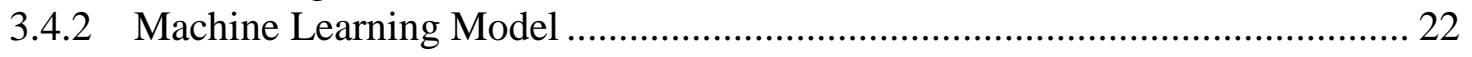

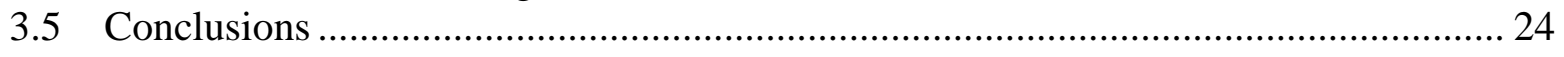

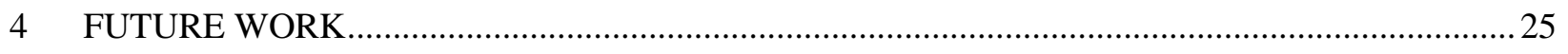

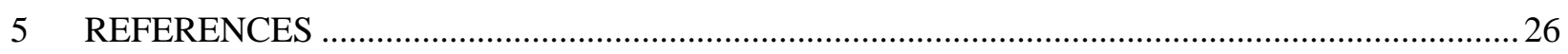

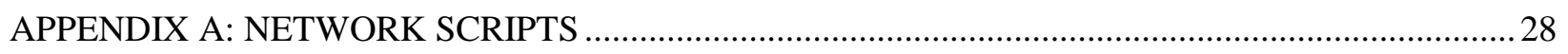

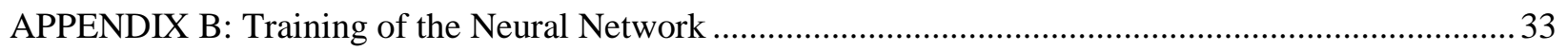




\section{FIGURES}

1-1 HTGR (a) normal operating condition, and (b) natural circulation pathways (yellow arrows) during a PCC event [1-2]. 1

2-1 Simplified model geometry for single coolant channel flow, with a (a) side view, and (b) visible view to show inlet and outlet. 5

2-2 Comparison of absolute errors for axial mean velocities between fine, medium, and coarse grid along the center of the single jet (relative errors are in the same order as absolute errors).

2-3 Comparison of velocities over measured plane (XY-plane) in Nek5000 for $\mathrm{Re}=12,819$; (i) axial mean velocity and (ii) velocity magnitude. Fluid properties are considered at a temperature of $292.74 \mathrm{~K}$.

2-4 Decay profiles of local velocity along the jet center-line for Re of 12,819. 10

2-5 Axial mean velocities across $x$-position at various axial locations; (i) $\operatorname{Re}=3,413$, (ii) $\operatorname{Re}=7,912$ and (iii) $\operatorname{Re}=12,819$. 10

2-6 Reynolds stress across $x$-position at various axial locations; (i) $\operatorname{Re}=3,413$, (ii) $\mathrm{Re}=7,912$, and (iii) $\mathrm{Re}=12,819$.

2-7 Axial velocity RMS fluctuations across $\mathrm{x}$-position at various axial locations;

(i) $\operatorname{Re}=3,413$, (ii) $\operatorname{Re}=7,912$, and (iii) $\operatorname{Re}=12,819$.

2-8 Radial velocity RMS fluctuations across $\mathrm{x}$-position at various axial locations;

(i) $\mathrm{Re}=3,413$, (ii) $\mathrm{Re}=7,912$, and (iii) $\mathrm{Re}=12,819$, numerical modeling. 13

3-1 Comparison of (i) Nek5000 (or) experimental data and Sieder-Tate Correlation for (ii) Nusselt number.

3-2 Architecture of a fully connected, deep feed-forward neural network................................... 19

3-3 Comparison of Nusselt number for model and Nek5000 data............................................ 22

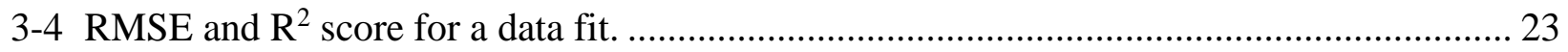

3-5 Direct comparison of DFNN predictions and original Nu.................................................. 23

3-6 Histogram of relative error of DFNN predictions................................................................ 24 


\section{TABLES}

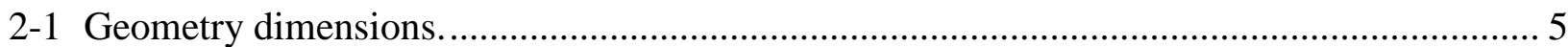

2-2 Operating conditions and thermo-physical properties for the single coolant channel configuration.

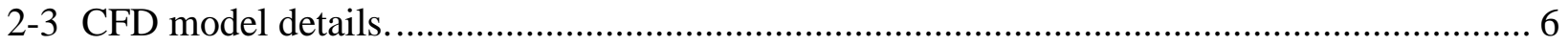

2-4 Normalized errors between simulations and experiments for axial velocity. ......................... 7

2-5 Normalized errors between simulations and experiments for axial root-mean-square (RMS) of axial (v') velocity................................................................... 7

2-6 Normalized errors between simulations and experiments for RMS of x-component (u') velocity................................................................................................... 7

2-7 Normalized errors between simulations and experiments for Reynolds stress (u'v')............. 7

2-8 Mesh information for grid independence study. ................................................................. 8

3-1 DTHT benchmark test operating conditions for inlet temperature of $304 \mathrm{~K}$......................... 16

3-2 Description of various heat-transfer parameters. …………………………………............. 17

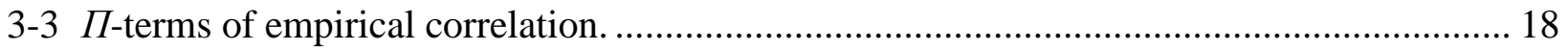




\section{ACKNOWLEDGMENTS}

This work was primarily funded by the U.S Department of Energy, Office of Nuclear Energy, Nuclear Energy Advanced Modeling and Simulation (NEAMS) program. It was also supported by Laboratory Directed Research and Development (LDRD) funding from Argonne National Laboratory, provided by the Director, Office of Science, of the U.S. Department of Energy under Contract No. DE-AC02-06CH11357.

We gratefully acknowledge the computing resources provided on Bebop and Mira, a highperformance computing cluster operated by the Laboratory Computing Resource Center (LCRC) and Argonne Leadership Computing Facility (ALCF) at Argonne National Laboratory. 


\section{ACRONYMS AND ABBREVIATIONS}

1D, 2D, 3D One-, two-, three-dimensional

$\begin{array}{ll}\text { ANN } & \text { artificial neural network } \\ \text { ASME } & \text { American Society of Mechanical Engineers } \\ \text { CFD } & \begin{array}{l}\text { computational fluid dynamics } \\ \text { CFL }\end{array} \\ \text { Courant-Friedrichs-Lewy } \\ \text { DCC } & \begin{array}{l}\text { depressurized conduction cooldown } \\ \text { deep feed-forward neural network }\end{array} \\ \text { DOE } & \begin{array}{l}\text { U.S. Department of Energy } \\ \text { DTHT }\end{array} \\ \text { deteriorated turbulent heat transfer } \\ \text { GCR } & \text { gas-cooled reactor } \\ \text { HTGR } & \text { high-temperature gas-cooled reactor } \\ \text { LES } & \text { Large Eddy Simulation } \\ \text { LOFC } & \text { loss of forced circulation } \\ \text { MHTGR } & \text { modular high-temperature gas-cooled reactor } \\ \text { MIT } & \text { Massachusetts Institute of Technology } \\ \text { ML } & \text { machine learning } \\ \text { MSE } & \text { mean square error } \\ \text { NE } & \text { Office of Nuclear Engineering (DOE) } \\ \text { NEAMS } & \text { Nuclear Energy Advanced Modeling and Simulation (DOE-NE) } \\ \text { PCC } & \text { pressurized conduction cooldown } \\ \text { RCCS } & \text { reactor cavity cooling system } \\ \text { ReLU } & \text { rectified linear units } \\ \text { RMS } & \text { root-mean-square } \\ \text { RMSE } & \text { root-mean-square error } \\ \text { RPV } & \text { reactor pressure vessel } \\ \text { SAM } & \text { system analysis module } \\ \text { TAMU } & \text { Texas A\&M University } \\ \text { VHTR } & \text { very-high-temperature gas-cooled reactor } \\ & \end{array}$




\section{NOTATION}

$\begin{array}{ll}\mathrm{C}_{\mathrm{p}} & \text { Specific heat }[\mathrm{J} / \mathrm{kg}-\mathrm{K}] \\ \mathrm{D}, \mathrm{d} & \text { Tube diameter }[\mathrm{m}] \\ \mathrm{L} & \text { Tube Length }[\mathrm{m}] \\ \mathrm{G} & \text { Mass flux }\left[\mathrm{kg} / \mathrm{m}^{2}-\mathrm{s}\right] \\ \mathrm{h} & \text { Heat transfer coefficient }\left[\mathrm{W} / \mathrm{m}^{2}-\mathrm{K}\right] \\ \mathrm{q} & \text { Heat flux }\left[\mathrm{w} / \mathrm{m}^{2}\right] \\ \mathrm{k} & \text { Thermal conductivity }[\mathrm{W} / \mathrm{m}-\mathrm{K}] \\ \mathrm{Nu} & \text { Nusselt number }[-] \\ \mathrm{Re} & \text { Reynolds number }[-] \\ \mathrm{Pe} & \text { Peclet number }[-] \\ \mathrm{Pr} & \text { Prandtl number }[-] \\ \mathrm{u} & \text { Velocity }[\mathrm{m} / \mathrm{s}] \\ \mu & \text { Viscosity }[\text { Pa-s] } \\ \mathrm{z} & \text { Axial local length }[\mathrm{m}] \\ \rho & \text { Density }\left[\mathrm{kg} / \mathrm{m}^{3}\right]\end{array}$

Subscripts

$\begin{array}{ll}\text { b } & \text { bulk } \\ \text { w } & \text { wall } \\ \text { exp } & \text { experiment } \\ \text { sim } & \text { simulation }\end{array}$




\section{EXECUTIVE SUMMARY}

With support from the U.S. Department of Energy-Office of Nuclear Energy's (DOENE's) Nuclear Energy Advanced Modeling and Simulation (NEAMS) program, an effort has been pursued to support high-temperature gas-cooled reactor (HTGR) technology development and its modeling and simulation needs. There is a particular need for advanced modeling and simulation tools to predict thermal-fluid behavior in the nuclear reactor primary system, especially in the core and the lower and upper plena, during safety-related transients. In this report, two main such activities are presented relevant to the HTGRs: (1) three-dimensional (3D) computational fluid dynamics (CFD) validation using benchmark data from the upper plenum of Texas A\&M University's 1/16th scaled very-high-temperature gas-cooled reactor (VHTR), and (2) development of wall heat-transfer correlation for laminar flow in a wall-heated pipe.

The CFD tool validation exercises can be helpful to choose the models and CFD tools to simulate and design specific components of the HTRGs such as upper plenum where jet mixing is a complex phenomenon. In a loss of forced circulation event, the laminar flow can be observed during the development of natural circulation flow. This work includes the development and validation of heat transfer correlations for laminar flow using the Nek5000 CFD code due to limited available experimental data for laminar flow conditions to guide low-order models (1D).

In this report, the flow characteristics of a single isothermal jet discharging into the upper plenum was investigated using the Nek5000 Large-Eddy Simulation (LES) CFD tool. Several numerical simulations were performed for various jet-discharged Reynolds numbers ranging from 3,413 to 12,819. A grid-independent study was performed. The numerical results of mean velocity, root-mean-square fluctuating velocity, and Reynolds stress were compared against the benchmark data. Good agreement was obtained between simulated and measured data for axial mean velocities, except near the upper plenum hemisphere. The maximum predicted errors for axial mean velocities at various normalized coolant channel diameter heights of 1,5 , and 10 are $1.56 \%$, $1.88 \%$, and $3.82 \%$, respectively. In addition, the predicted root-mean-square fluctuating velocity and Reynolds stress are qualitatively in agreement with the experimental data.

The Nek5000 code was used to develop wall-heat transfer correlation for laminar flow in a cylindrical tube. Several simulations were performed for various Reynolds flow and wall-heat fluxes. A new heat transfer correlation was developed using data from Nek5000 simulation results and regression functions in Matlab. The developed heat transfer correlation is valid for various Reynolds flows from 200 to 2000. The predicted $\mathrm{R}^{2}$ value for model fit was 0.875 , which ensures that $87.5 \%$ of the model data lies on the Nek5000 data. Moreover, a machine learning (ML) tool was used to train and test the Nek5000 data. A good fit of the ML-based model was observed with the test data. 
This page is intentionally left blank. 


\section{INTRODUCTION}

For safety and licensing purposes, we must analyze the transient response of hightemperature gas-cooled reactors (HTGRs) to hypothetical accident scenarios such as loss of forced circulation (LOFC) following a loss-of-offsite power event. Depending on whether there is a leak in the primary pressure boundary, the accident scenario is commonly referred to as a pressurized conduction cooldown (PCC) or a depressurized conduction cooldown (DCC) event. In either case, the downward core flow under conditions of normal operation (Figure 1-1(i)) reverses and flows upward (Figure 1-1(ii)), resulting in a complex flow and temperature distributions in the core. During PCC or DCC, it is a safety concern that the very hot coolant flow leaving the core region could form jets and then impinge on the upper plenum structures. This introduces undesirable temperature gradients in the structures and leads to material cracking. Moreover, there is a potential for the heat transfer to be degraded during the transition to natural circulation as coolant in the channels may shift from a turbulent to a laminar flow regime.

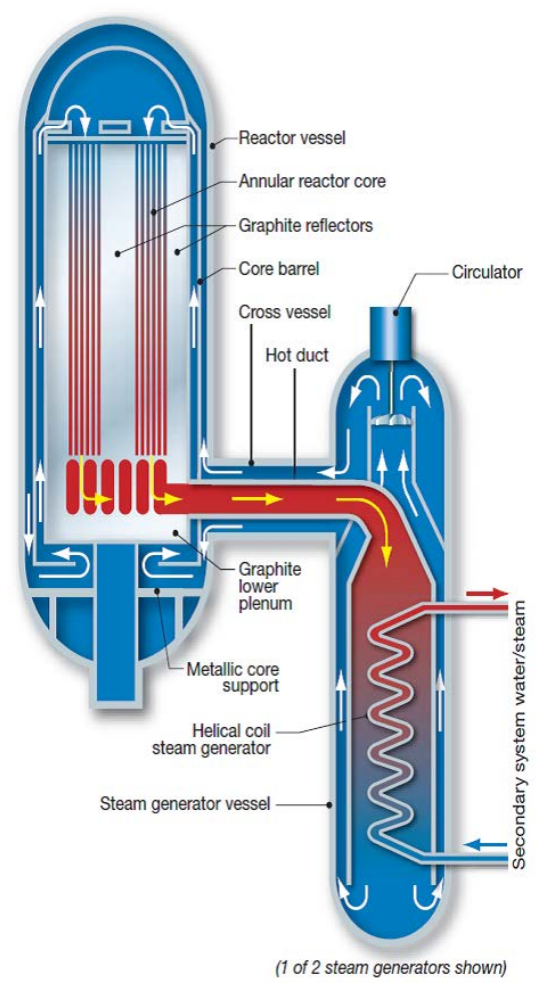

(i)

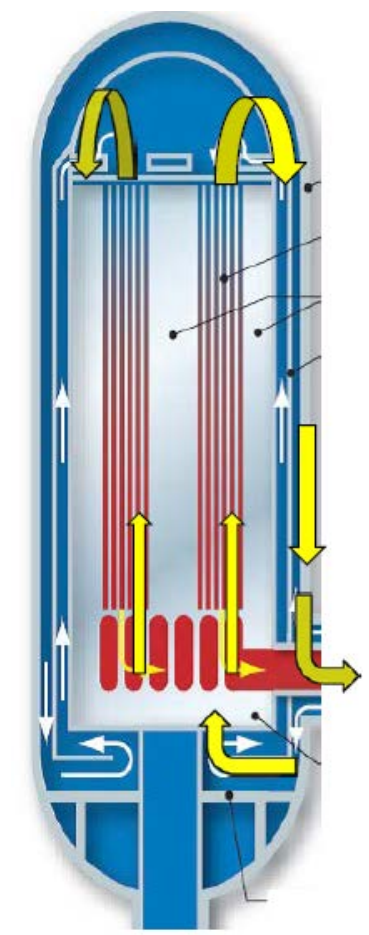

(ii)

FIGURE 1-1 HTGR (i) normal operating condition, and (ii) natural circulation pathways (yellow arrows) during a PCC event [1-2] 
Assumptions for the postulated LOFC scenario include: the initiating event leads to a loss of offsite power, a reactor scram, and thus a loss of power to the blower. The scenario quickly progresses from the system's normal operational conditions to a natural circulation condition as the blower coast down. The reactor flow, normally moving downward through the core and into the lower plenum, stagnates and reverses because the driving force is the helium density gradient in the primary heat transport system. Hence, the flow occurs upward through the core and forms jets and plumes that leave the top of the core and may move upward to impinge on the ceiling of the upper plenum. Heat transfer within the core moves radially through the prismatic blocks, while some fraction of the core-generated heat is carried upward by the natural circulation flow. The graphite in the core and the core reflectors, nominally cool, begin a prolonged heat-up (the graphite acts as a heat-storage medium) while heat is transferred through the reactor vessel walls to be transferred both from the reactor vessel walls to the reactor cavity cooling system (RCCS) via radiation and to the confinement air via convective currents driven by convective heat transfer. The heat transferred to the RCCS is transported to the environment via the RCCS cooling channels, which deliver environmental air to the RCCS ducts to pick up heat and to transport the heat energy to the environment. Heat transfer to the medium surrounding the containment structure-soilgiven that the modular HTGR [MHTGR] is installed below ground-also assists in removing heat from the containment. It should be noted that RCCS behavior is generally very important in LOFC events because the RCCS becomes the only effective means of removing afterheat from the core and vessel.

The core coolant flow distribution during the LOFC is dictated by a number of factors, such as (1) the core power distribution; (2) helium viscous properties, namely, helium viscosity increases with increasing temperature: hence, hot spots have reduced helium cooling for unchanging pressure but increasing temperature conditions, whereas cool spots have increased helium cooling stemming from lower temperatures relative to the hot spots; and (3) the core bypass fraction. The presence of adequate core cooling will ensure that peak fuel temperatures do not approach the regulatory limits for upper temperatures. Factors that are important for ensuring that fuels and structural materials in an operational gas-cooled reactor (GCR) do not exceed a regulatory limit include the following:

i. Localized core cooling distributions proportional to the magnitude of localized core power; that is, if the design is predicated on ensuring that adequate cooling is used to assure adequate cooling for "hot" fuel pins, then the core fuel bulk temperatures will always be well below the regulatory limits for allowable peak fuel temperatures.

ii. Prevention or proper accounting for effects that reduce core cooling, such as laminarization, a degraded turbulent heat-transfer phenomena.

The GCR designs studied to date generally follow the practice given as item (i) above. However, the level-of-knowledge requirement does not include convincing evidence that phenomena such as laminarization will not occur. For some natural circulation conditions, experiments performed at the Massachusetts Institute of Technology (MIT) have demonstrated the presence of laminarization [3] under certain conditions; further clarification is needed as to whether such conditions will occur in an HTGR. For the core, during operational and accident 
conditions, the core channel flow rates in a prismatic GCR may be laminar, turbulent, or in a transition region. If the flow is turbulent, there is the possibility that laminarization exists.

The bypass flows in a prismatic GCR are of concern because they reduce the desired flow rates in the coolant channels and, thereby, can increase outlet gas temperatures and maximum fuel temperatures. In the existing literature, bypass flows of 10 to $30 \%$ of the total flow rate have been estimated [1]. Consequently, it is important to account for bypass flows in analyses of reactor thermal gas dynamics. Hence, the bypass flow effects in HTGRs should be addressed in future work.

In our previous work, MHTGR full-loop simulations were performed using system analysis module (SAM) code under both normal and accident operating conditions [4]. Two approaches were used to model the reactor core: (1) the unit-cell or lumped parameter model, and (2) the ring model for all reactor core components, including reflectors, the reactor pressure vessel (RPV), and the RCCS. Both approaches include the lower and upper plenum, heat exchanger, and blower components for primary loop simulations. In normal operating conditions, the calculated temperatures were within the designed limits and obtained good agreement with analytical values. In accident scenarios, the natural circulation flow was developed due to loss of forced circulation flow and heat exchanger failure. The significant temperature increase was observed in heat structures (fuel rings and reflectors) and the coolant.

In our previous work, CFD models were validated with the MIT benchmark test [5]. In the current work, CFD models were validated with the Texas A\&M University (TAMU) $1 / 16^{\text {th }}$ scaled VHTR benchmark test [6-7]. Further, the Nek5000 LES model was used to develop the wall heattransfer correlations due to limited available experimental data for laminar flow conditions to guide low-order models like SAM [8], Pronghorn [9], etc.

In this report, 3D CFD models were validated with the TAMU $1 / 16^{\text {th }}$ Scaled VHTR benchmark test as described in Section 2. In Section 3, 3D CFD models were used to develop the wall heat-transfer correlations. Finally, future work was proposed in Section 4. 


\section{CFD MODEL VALIDATION FOR A BENCHMARK DATA OF TEXAS A\&M UNIVERSITY $1 / 16^{\text {th }}$ SCALED VHTR UPPER PLENUM}

\subsection{BACKGROUND}

Advanced computational tools can help evaluate the sensitivity of the behavior of the hot plumes to reactor design parameters like channel geometry, number of channels, and heating profiles under hypothetical accident scenarios such as LOFC. One of the first steps in developing such an advanced capability is to validate existing CFD tools for analysis of jet mixing in geometries similar to that of the upper plenum. In this work, CFD code Nek5000 [10] was used to analyze the $1 / 16^{\text {th }}$ scaled single-jet VHTR upper plenum experiments performed at Texas A\&M University [6]. In these experiments, fluid enters a lower plenum from a horizontal inlet pipe, flows into a single vertical inlet channel, and enters the scaled dome-shaped test section where the jet mixing occurs [6]. In fiscal year 2019 (FY 2019), the ASME V\&V 30 subcommittee (Verification and Validation in Computational Nuclear System Thermal Fluids Behavior Committee) selected the $1 / 16^{\text {th }}$ scaled single-jet VHTR upper plenum for CFD models validation to study the flow mixing of a single-jet isothermal flow in the upper plenum.

In this report, the simulation results were analyzed for flows with various Reynolds numbers (Re) from 3,413 to 12,819 in a single coolant channel configuration. Several numerical simulations were performed to validate the numerical models with experimental data.

\subsection{MODELED GEOMETRY}

As shown in Figure 2-1, the jet flow is allowed in a single coolant channel, and the remaining 24 channels were closed (not shown). The opened channel is at the center of all of the coolant channels and is highlighted in Figure 2-1(ii). In this configuration, both inlet and outlet channels were simplified due to the negligible influence on jet velocities in the upper plenum. The details of the experimental facility, operating conditions, and measured data are provided in Alwafi et al. [6]. The measurements of jet velocity and flow distributions within the upper plenum were performed using time-resolved particle image velocimetry for various jet Reynolds numbers $(3,413$ to 12,819). In numerical simulations, the lower plenum was not considered. This simplification is valid because of the single vertical inlet channel where total mass flow rate enters from the lower plenum. In addition, the recycling boundary condition was applied to generate fully developed flow in a long vertical pipe (coolant channel) as shown in Figure 2-1(i). 


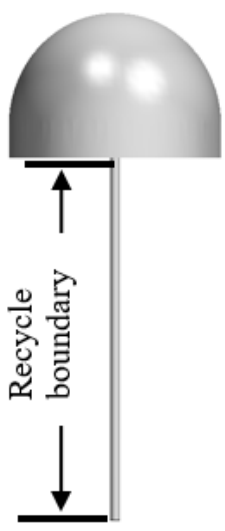

(i)

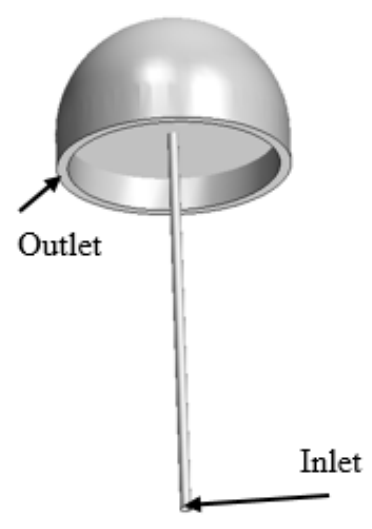

(ii)

FIGURE 2-1 Simplified model geometry for single coolant channel flow, with a (i) side view, and (ii) visible view to show inlet and outlet.

TABLE 2-1 Geometry dimensions.

\begin{tabular}{ll}
\hline Plenum diameter [m] & 0.4083 \\
Inner core diameter [m] & 0.37 \\
Upper plenum height [m] & 0.197 \\
Coolant channel diameter [m] & 0.01905 \\
Number of coolant channels & 1 \\
Coolant channel height [m] & 0.7749 \\
Lower plenum depth [m] & 0.05625 \\
Lower plenum diameter [m] & 0.37 \\
\hline
\end{tabular}

TABLE 2-2 Operating conditions and thermo-physical properties for the single coolant channel configuration.

\begin{tabular}{ll}
\hline Inlet Reynolds number $(\mathrm{Re})$ & $3,413,7,912,12,819$ \\
Inlet temperature $[\mathrm{K}]$ & 292.89 \\
Water viscosity $[\mathrm{Pa} \cdot \mathrm{s}]$ & 0.001008 \\
Water density $\left[\mathrm{kg} / \mathrm{m}^{3}\right]$ & 998.26 \\
Outlet pressure $[\mathrm{Pa}]$ & 0.0 \\
\hline
\end{tabular}


TABLE 2-3 CFD model details.

\begin{tabular}{|l|l|}
\hline 3D simulations & Isothermal transient \\
Numerical method & Spectral element \\
Turbulence & LES \\
Working fluid & Water \\
$\begin{array}{l}\text { Mesh, no. of Gauss-Legendre-Lobatto } \\
\text { (GLL) points [Million] }\end{array}$ & 213 \\
Order of numerical accuracy & \\
Courant-Friedrichs-Lewy (CFL) & 2 \\
\hline
\end{tabular}

\subsection{NUMERICAL MODELING}

The 3D CFD isothermal LESs were performed using Nek5000. The simulated geometry details and operating conditions are shown in Tables 2.1 and 2.2. The inlet velocity is computed based on specified Reynolds number, coolant channel diameter, working fluid density, and viscosity. The Nek5000 uses the spectral element method (SEM) to solve the Navier-Stokes fluid flow equations numerically. The merit of Nek5000 is that it is a highly scalable, open source, transient CFD code, and it has run on more than 1 million processors on Argonne's massively parallel Blue Gene/Q computer, Mira [10]. The velocity equations were solved using an incompressible, transient, isothermal, Helmholtz solver [10]. In this work, simulations were performed until the specified convergence criteria were met (normalized residual is $10^{-5}$ ).

For this validation study, hexahedral mesh was generated for Nek5000 simulations in the Ansys ICEM CFD tool, and the full 3D volume was discretized with 213 million Gauss-LegendreLobatto (GLL) points with a minimum GLL point size of $0.1 \mathrm{~mm}$ in the jet region.

A pressure boundary condition was specified at the outlet, flow velocity was specified at the inlet, and a no-slip boundary condition was imposed at the wall. The wall-resolved LES was used in Nek5000. In geometry, the maximum wall cell height is $0.025 \mathrm{~mm}$ to ensure that $\mathrm{y}+$ is less than one. In addition, the recycle boundary condition was applied to generate fully developed flow in the long vertical pipe, as discussed above [5]. In the recycle boundary, the velocity components $(\mathrm{u}, \mathrm{v}, \mathrm{w})$ of the predicted upper plenum inlet (the outlet of the coolant channel) are recycled (or) assigned to the coolant channel inlet at each numerical time step, and that treatment also ensures (or) enforced mass conservation (Figure 2-1a).

In LES, a low-pass filtering technique was applied to Navier-Stokes equations to eliminate small scales of the solution in order to reduce the computational cost of the simulation. By doing so, the solution obtained is a filtered flow field [10]. It is of note that the LES formulation relies on slight (8\%) filtering of modes $\mathrm{k}=\mathrm{N}-1$ and $\mathrm{N}$, which provides an energy drain at the unresolved grid scale. The numerical solution is second-order accurate as shown in Table 2-3. Tables 2-4 through 2-7 show values for normalized errors between simulations and experiments under various conditions.

$$
\operatorname{Error}(\%)=\frac{\sqrt{\sum_{i=1}^{n}\left(\exp _{i}-\operatorname{sim}_{i}\right)^{2}}}{\sqrt{\sum_{i=1}^{n} \exp _{i}^{2}}}
$$


TABLE 2-4 Normalized errors between simulations and experiments for axial velocity.

\begin{tabular}{lccc}
\hline & \multicolumn{3}{c}{ Error (\%) } \\
\cline { 2 - 4 } $\begin{array}{c}\text { Reynolds } \\
\text { number }\end{array}$ & $\mathrm{y} / \mathrm{d}=1$ & $\mathrm{y} / \mathrm{d}=5$ & $\mathrm{y} / \mathrm{d}=10$ \\
\hline & & & \\
3,413 & 1.56 & 1.88 & 3.57 \\
7,912 & 0.83 & 0.93 & 3.22 \\
12,819 & 0.69 & 1.28 & 3.82 \\
\hline
\end{tabular}

TABLE 2-5 Normalized errors between simulations and experiments for axial root-mean-square (RMS) of axial (v') velocity.

\begin{tabular}{llll}
\hline & \multicolumn{3}{c}{ Error (\%) } \\
\cline { 2 - 4 } $\begin{array}{c}\text { Reynolds } \\
\text { number }\end{array}$ & $\mathrm{y} / \mathrm{d}=1$ & $\mathrm{y} / \mathrm{d}=5$ & $\mathrm{y} / \mathrm{d}=10$ \\
\hline & & & \\
3,413 & 6.18 & 3.56 & 3.06 \\
7,912 & 2.64 & 5.06 & 4.11 \\
12,819 & 4.84 & 5.05 & 5.60 \\
\hline
\end{tabular}

TABLE 2-6 Normalized errors between simulations and experiments for RMS of $x$-component ( $u^{\prime}$ ) velocity.

\begin{tabular}{lccc}
\hline & \multicolumn{3}{c}{ Error (\%) } \\
\cline { 2 - 4 } $\begin{array}{c}\text { Reynolds } \\
\text { number }\end{array}$ & $\mathrm{y} / \mathrm{d}=1$ & $\mathrm{y} / \mathrm{d}=5$ & $\mathrm{y} / \mathrm{d}=10$ \\
\hline & & & \\
3,413 & 5.73 & 1.67 & 3.07 \\
7,912 & 4.52 & 6.47 & 3.62 \\
12,819 & 6.85 & 5.57 & 3.34 \\
\hline
\end{tabular}

TABLE 2-7 Normalized errors between simulations and experiments for Reynolds stress (u'v').

\begin{tabular}{lccc}
\hline & \multicolumn{3}{c}{ Error (\%) } \\
\cline { 2 - 4 } $\begin{array}{c}\text { Reynolds } \\
\text { number }\end{array}$ & $\mathrm{y} / \mathrm{d}=1$ & $\mathrm{y} / \mathrm{d}=5$ & $\mathrm{y} / \mathrm{d}=10$ \\
\hline & & & \\
3,413 & 15.3 & 41.6 & 176.5 \\
7,912 & 24.3 & 51.0 & 172.9 \\
12,819 & 41.44 & 35.0 & 257.8 \\
& & & \\
\hline
\end{tabular}




\subsection{GRID INDEPENDENCE STUDY}

Three different meshes were studied to justify the grid independence on jet axial mean velocities for a Reynolds number of 12,819 (Table 2-8). As Figure 2-2 shows, the calculated absolute errors for axial mean velocities along the center of the jet are consistent in both fine and medium mesh due to low magnitudes. In contrast, significant deviations were found for a coarse mesh. The predicted slope for power spectral density (PSD) was $-5 / 3$, which ensures that the decay of turbulence in the inertial sub-range is accurate (not shown). Both the grid independence study and turbulence decay rate conclude that the medium coarse grid is good enough to validate the CFD models for $\mathrm{Re}=12,819$. Hence, the simulations were performed on the medium coarse grid for all runs as shown in Figure 2-2. In the following section, the results presented are grid independent.

TABLE 2-8 Mesh information for grid independence study.

\begin{tabular}{ll}
\hline Mesh & GLL points \\
Coarse & $416046 \times 7^{3}$ \\
Medium & $416046 \times 8^{3}$ \\
Fine & $416046 \times 9^{3}$ \\
\hline
\end{tabular}

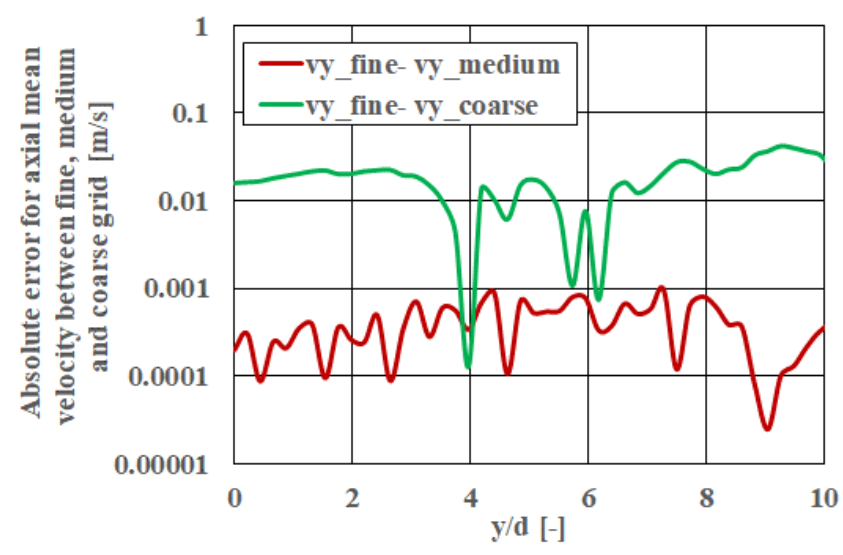

FIGURE 2-2 Comparison of absolute errors for axial mean velocities between fine, medium, and coarse grid along the center of the single jet (relative errors are in the same order as absolute errors). 


\subsection{RESULTS}

In this section, three different single-jet experiments were modeled and simulated for given operating conditions as shown in Tables 2-1 and 2-2. Nek5000 simulations were performed and analyzed for various Reynolds flows from 3,413 to 12,819. As Figure 2-1 shows, a single channel is considered at the center of the core or upper plenum. It is of note that the Nek5000 time-steps were limited based on a CFL of 0.5. Hereafter, the presented numerical results are statistically steady state.

As Figure 2-3 shows, the jet reaches to the upper plenum hemisphere where jet impingement may lead to hot spots on the wall surface in non-isothermal flows. Similarly, observations were found for other Reynolds number flows (3,413 and 7,912). In high Reynolds number flows ( $\operatorname{Re}>3,413)$, multiple recirculation zones were observed in the off-side of the jet. In the gas-cooled reactors, these recirculation zones are undesirable where hot or cold fluids can be trapped, leading to high thermal gradients in non-isothermal flows. In this paper, CFD models are validated with the experimental data at various axial locations, as shown in Figure 2-3 (where $\mathrm{y} / \mathrm{d}=1,5$, and 10$)$.

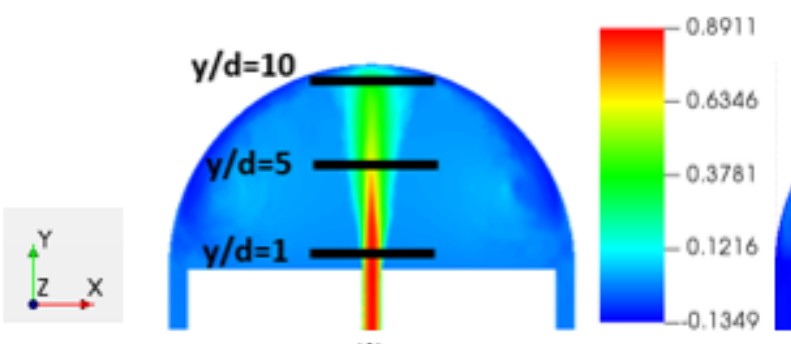

(i)

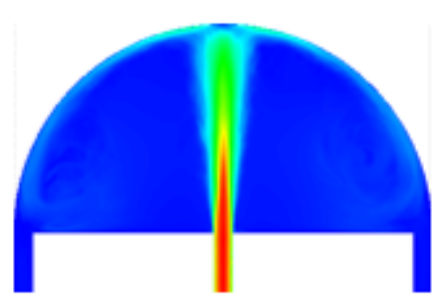

(ii)

FIGURE 2-3 Comparison of velocities over measured plane (XY-plane) in Nek5000 for $R e=12,819$; (i) axial mean velocity and (ii) velocity magnitude. Fluid properties are considered at a temperature of $292.74 \mathrm{~K}$.

Figure 2-4 illustrates the decay rate of the center-line velocity for Re of 12,819. The decay rate is defined as the ratio of centerline jet velocity ( $\left.\mathrm{V}_{\text {centerline}}\right)$ and maximum axial velocity (Vc). The decay rate is constant up to $\mathrm{y} / \mathrm{d}=3$. Further downstream, the decay rate is increased by two factors from 3 to 9 . Near the upper plenum hemisphere, the decay rate exponentially increases due to the jet impingement. 


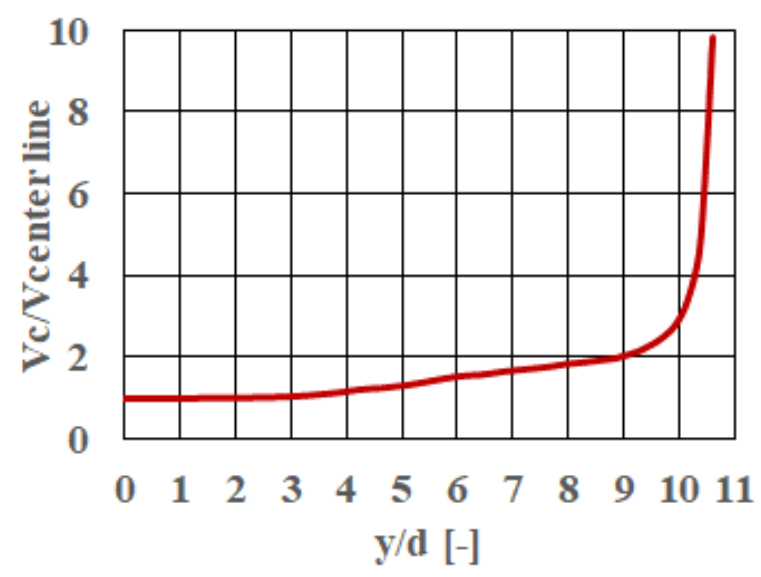

FIGURE 2-4 Decay profiles of local velocity along the jet center-line for $\operatorname{Re}$ of 12,819.

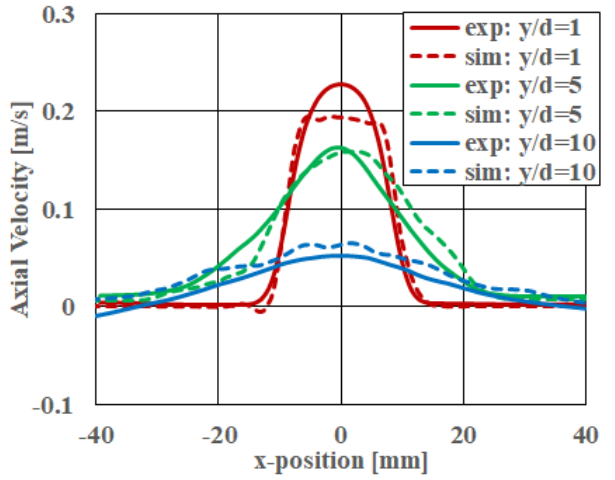

(i)

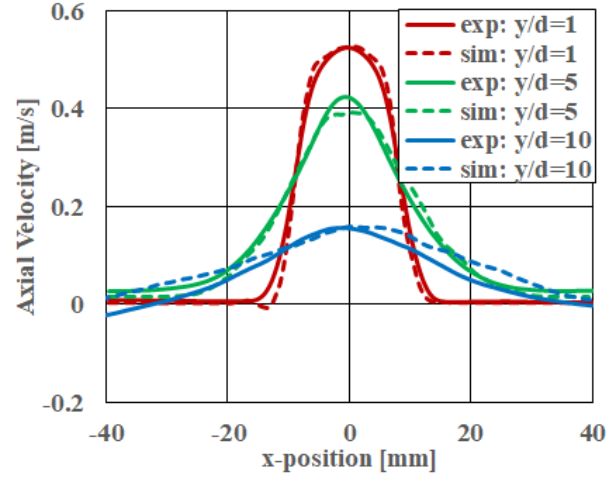

(ii)

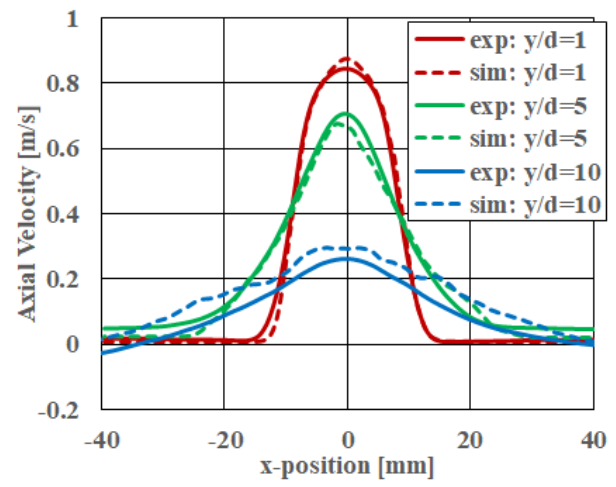

(iii)

FIGURE 2-5 Axial mean velocities across $x$-position at various axial locations; (i) $\operatorname{Re}=3,413$, (ii) $\operatorname{Re}=7,912$ and (iii) $\operatorname{Re}=12,819$. 
In Figure 2-5, the axial mean velocities are shown for various Reynolds number flows. Both experimental and simulation data agreed qualitatively and quantitatively. Good agreement was observed for velocity peaks except near the upper plenum inlet $(y / d=1)$ for low $\operatorname{Re}(3,413)$. This result is due to lower viscosity ( 0.01 factor of molecular viscosity), which was used to generate the turbulence in the inlet pipe. For high Re, the molecular viscosity was used everywhere including for both the inlet pipe and upper plenum. Near the upper plenum hemisphere $(\mathrm{y} / \mathrm{d}=10)$, the overpredicted velocities were observed for all Reynolds flows. This result may occur due to an applied, no-slip velocity boundary condition for the hemisphere wall. The partial slip at the wall may lead to jet dispersion similar to the case of the experimental data. The predicted errors, as defined in equation (1), for axial mean velocities were shown in Table 2-4. The predicted error values are $3.82 \%$. The measurement uncertainties for velocities, root-mean-square (RMS) velocity fluctuations, and Reynolds stress were reported as being around $<1 \%$ [5]. The jet spread rate increases toward the upper plenum hemisphere due to momentum exchange with the surrounding fluid.

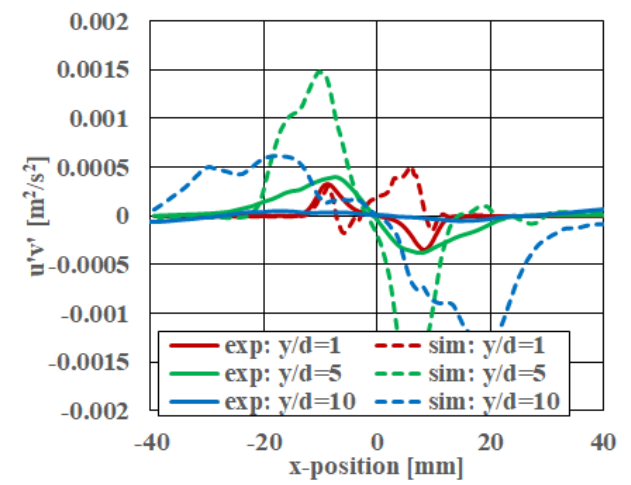

(i)

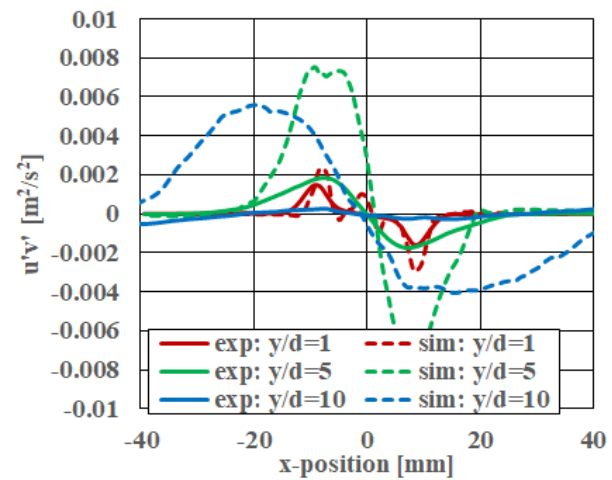

(ii)

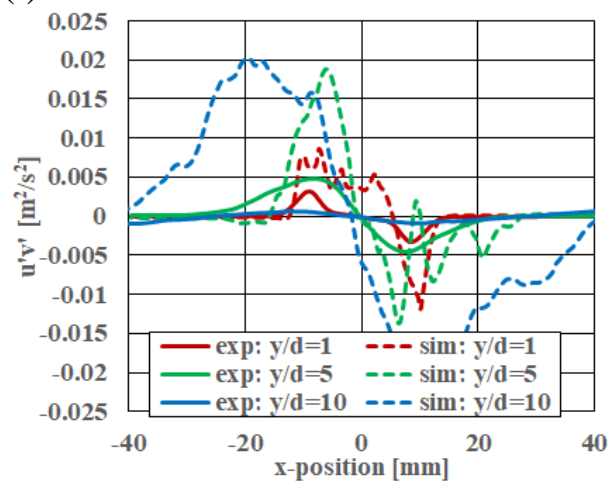

(iii)

FIGURE 2-6 Reynolds stress across $x$-position at various axial locations; (i) $\operatorname{Re}=3,413$, (ii) $\operatorname{Re}=7,912$, and (iii) $\operatorname{Re}=12,819$. 


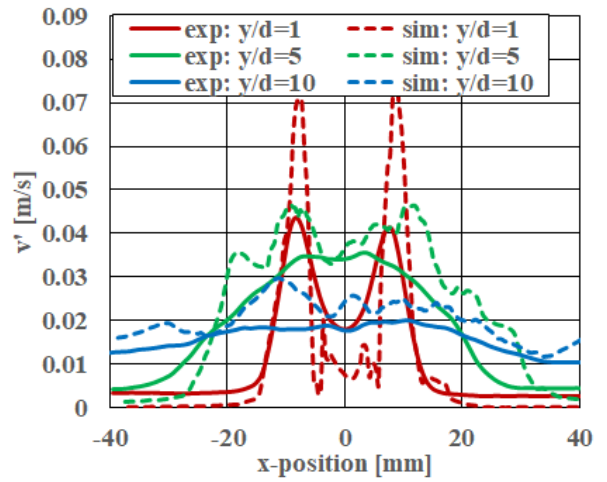

(i)

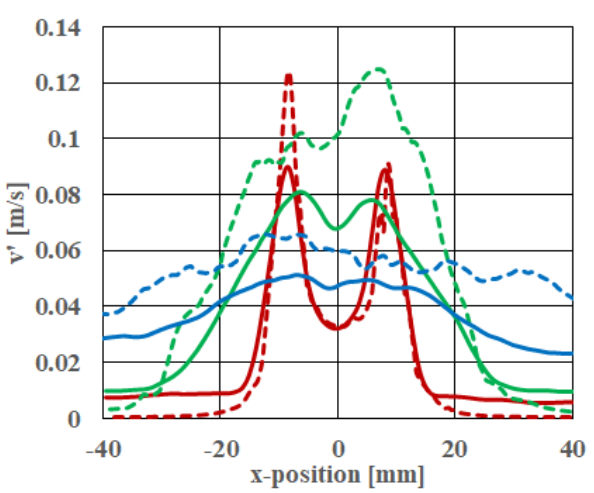

(ii)

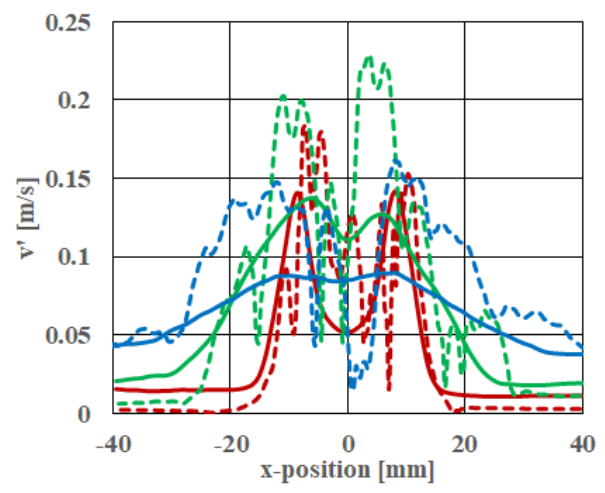

(iii)

FIGURE 2-7 Axial velocity RMS fluctuations across $x$-position at various axial locations; (i) $R e=3,413$, (ii) $R e=7,912$, and (iii) $R e=12,819$. 


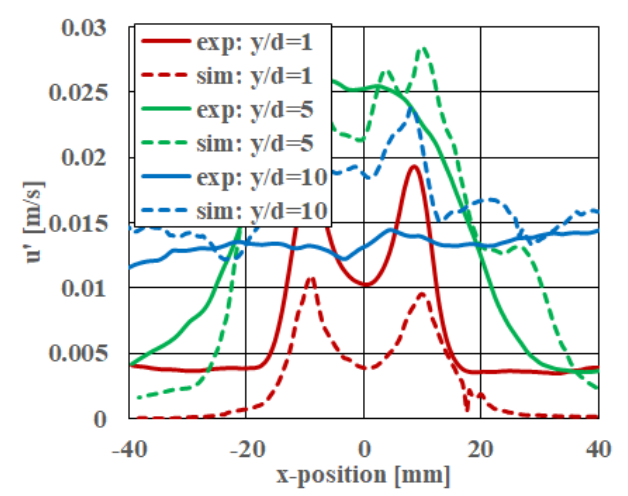

(i)

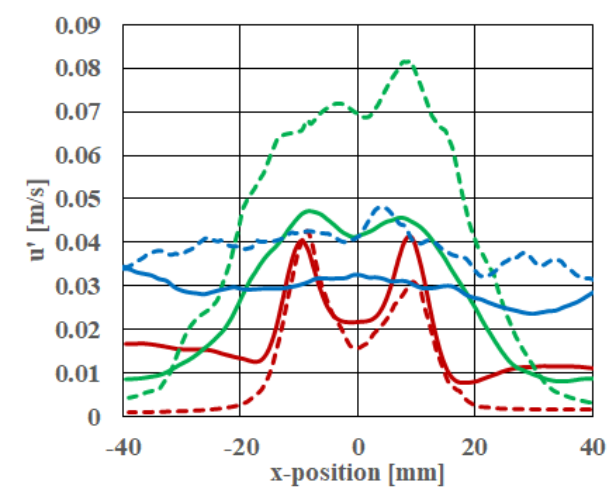

(ii)

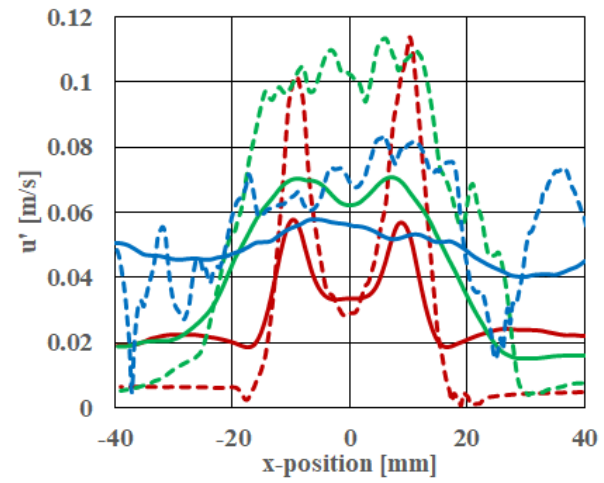

(iii)

FIGURE 2-8 Radial velocity RMS fluctuations across $x$-position at various axial locations; (i) $\operatorname{Re}=3,413$, (ii) $R e=7,912$, and (iii) $R e=12,819$, numerical modeling.

In Figures 2-6 to 2-8, Reynolds stress and RMS fluctuating velocities (axial- and radialcomponents) are shown. Although the Reynolds stress agreed qualitatively, it was quantitatively over-predicted. Similar observations were found for RMS velocity fluctuations of axial and radial components. The errors between simulations and experiments for velocity fluctuations are provided in Tables 2-5 through 2-7. In simulations, the axial mean velocity, RMS velocity fluctuations, and Reynolds stress deviations are possibly attributable to turbulence model.

In Figures 2-7 and 2-8, two peaks were observed for the RMS axial and radial fluctuating velocity, results that were similar to experimental data near the upper plenum inlet $(\mathrm{y} / \mathrm{d}=1)$. These peaks are located mainly over shear layers where jet momentum exchange occurs with surrounding fluid. Further downstream, the two peaks merged into a single peak as has occurred in experimental observations.

\subsection{CONCLUSIONS}

The 3D CFD simulations were performed to analyze the available experimental data for the upper plenum of the TAMU $1 / 16^{\text {th }}$ scale VHTR model. The Nek5000 LES code was used to simulate single jet flows with three different Reynolds numbers of 3,413, 7,912, and 12,819. Gridindependent study was performed for $\mathrm{Re}=12,819$. The predicted axial mean velocities, RMS 
velocity fluctuations, and Reynolds stresses were qualitatively in agreement with experimental data. Moreover, good agreement was observed for axial mean velocities for all Reynolds flows. The maximum predicted errors for axial mean velocities at various normalized coolant channel diameter heights of 1,5 , and 10 were $1.56 \%, 1.88 \%$, and $3.82 \%$, respectively. 


\section{DEVELOPMENT OF HEAT TRANSFER CORRELATIONS FOR LAMINAR FLOW IN A WALL-HEATED CYLINDRICAL TUBE}

\subsection{BACKGROUND}

Heat transfer from wall-to-fluid plays a significant role in gas-cooled reactors that carry heat via fluid. In an HTGR, the flow would be in the turbulent regime under normal operating conditions. In the literature, predictions of the wall temperatures with turbulent heat transfer correlation reported uncertainties of up to $\pm 20 \%$ [11]. Under accident scenarios, validation of the heat transfer correlation was shown in Lee et al [11]. Most of their data belongs to upward flow under hypothetical accident scenarios like PCC. We note that their experimental data are available for higher Reynolds numbers (Re>2000). The PCC occurred mainly as a result of to the loss-offorced-circulation due to blower trip-off. The deteriorated turbulent heat transfer (DTHT) phenomena were observed in their work due to buoyancy- and acceleration-driven flow. The uncertainty of the heat transfer coefficient was shown up to $70 \%[3,11]$.

In a PCC event, the laminar flow can be observed during the development of natural circulation flow. The Reynolds number can approach zero at an inflection point where the flow direction changes from forced downward to buyonacy-driven upward in coolant channels. Hence, the laminar flow plays a significant role. This work includes the development and validation of heat transfer correlations for laminar flow using the Nek5000 CFD code.

In our previous work [4], 3D CFD non-isothermal modeling and simulations were performed in a wall-heated circular tube. The simulations were verified with two different CFD tools, Nek5000 and STAR-CCM+, and validated with an experimental dataset (see Section 4 in Vegendla et al. [4]). The details of the geometry, modeling, and numerical convergence can also be found in Section 3 of [4]. In Nek5000 and STAR-CCM+, the predicted L2-norm values for wall temperature error (i.e., the difference between measured and simulation data) were $0.07 \%$ and $1.57 \%$, respectively. Hence, the Nek5000 code was selected to run simulations for extremely low Reynolds numbers (Table 3-1) to generate wall-heat transfer data (e.g., Nusselt number). 
TABLE 3-1 DTHT benchmark test operating conditions for inlet temperature of $304 \mathrm{~K}$.

\begin{tabular}{lrl}
\hline CASE & Re & \multicolumn{1}{c}{$\mathrm{q}_{\text {wall }}\left[\mathrm{W} / \mathrm{m}^{2}\right]$} \\
\hline $1-3$ & 200 & $2,000,5,000,7,000$ \\
$4-6$ & 400 &, \\
$7-9$ & 1,000 &, \\
$10-12$ & 2,000 &, \\
\hline
\end{tabular}

In our previous work [4], the mixed-convection heat transfer regime was observed for laminar flow. The heat transfer was increased up to $13 \%$ compared to fully developed flow; see Lee et al. [11] and Sieder-Tate correlations [12]. The Sieder-Tate correlation was valid only at tube entrance length (Figure 3-1i). Toward an outlet, the flow was not yet in a fully developed condition even though it was applied to a fully developed conditions at the inlet. This result was mainly attributable to flow profile modification (see Figure 3.8 in Vegendla et al. [4]) with the provided wall-heat flux condition. From Figure 3-1ii, the uncertainty of the heat-transfer coefficient (Nusselt) from the Sieder-Tate model was shown up to $\pm 60 \%$.

Sieder-Tate correlation equations [12] are as follows:

$$
\begin{gathered}
N u=1.86 \beta \\
\beta=\boldsymbol{P} \boldsymbol{e}^{\mathbf{1} / \mathbf{3}}\left(\frac{D}{L}\right)^{\mathbf{1 / 3}}\left(\frac{\mu_{\text {bulk }}}{\mu_{\text {wall }}}\right)^{\mathbf{0 . 1 4}}
\end{gathered}
$$

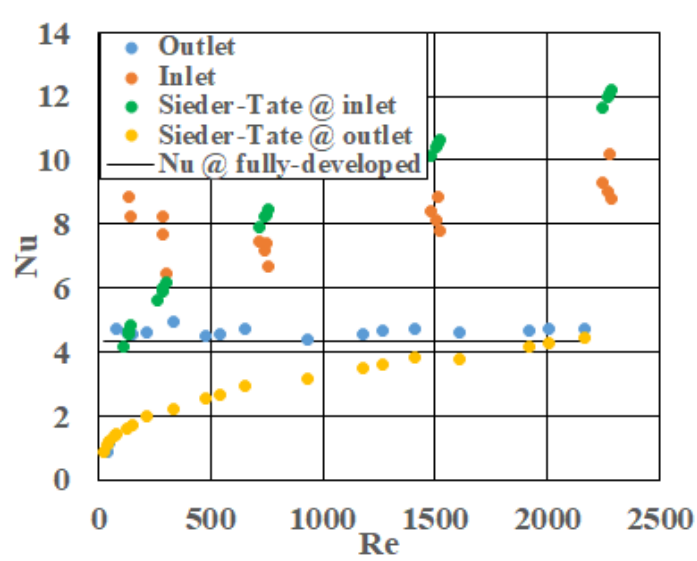

(i)

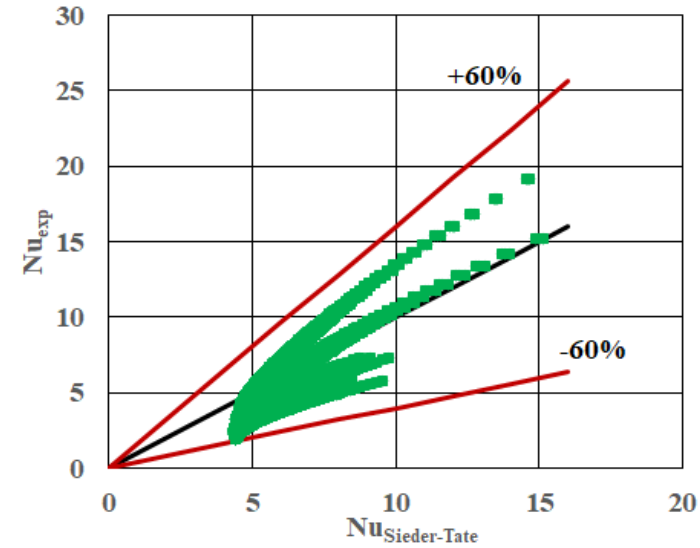

(ii)

FIGURE 3-1 Comparison of (i) Nek5000 (or) experimental data and Sieder-Tate Correlation for (ii) Nusselt number. 
In our current work, the main focus is on development of heat-transfer correlations for low Reynolds number $(\operatorname{Re}<2000)$ flows. A new set of heat-transfer correlations is proposed for laminar flow in the following section. The wall-heat transfer data (Nusselt number) from the Nek5000 simulations has been used to develop wall-heat transfer correlations. Two different approaches were selected to fit the data: Matlab regression and machine learning models.

\subsection{MATLAB REGRESSION MODEL}

In Matlab, the multiple linear regression function 'regress' was selected to perform correlation development. Regression models describe the relationship between a response (output) variable and one or more predictor (input) variables. Statistics allow us to fit linear, generalized linear, and nonlinear regression models, including stepwise models and mixed-effects models. Once we fit a model, it can be used to predict or simulate responses; assess the model fit using hypothesis tests; or use plots to visualize diagnostics, residuals, and interaction effects. Table 3-2 provides various heat-transfer parameters used in the Matlab regression.

TABLE 3-2 Description of various heat-transfer parameters.

\begin{tabular}{clll}
\hline Variable & \multicolumn{1}{c}{ Description } & SI Units & \multicolumn{1}{c}{ Dimensions (M,L,T,K) } \\
\hline h & Heat transfer coefficient & $\mathrm{W} / \mathrm{m}^{2}-\mathrm{K}$ & $\mathrm{MT}{ }^{-3} \mathrm{~K}^{-1}$ \\
$\mathrm{z}$ & Axial local length & $\mathrm{m}$ & $\mathrm{L}$ \\
$\mathrm{D}$ & Inside diameter of tube & $\mathrm{m}$ & $\mathrm{L}$ \\
$k_{b}$ & Thermal conductivity of fluid at $T_{\mathrm{b}}$ & $\mathrm{W} / \mathrm{m}-\mathrm{K}$ & $\mathrm{MLT}-3 \mathrm{~K}^{-1}$ \\
$\rho_{b}$ & Density of fluid at $T_{\mathrm{b}}$ & $\mathrm{kg} / \mathrm{m}^{3}$ & $\mathrm{ML}^{-3}$ \\
$\mu_{b}$ & Viscosity of fluid at $T_{\mathrm{b}}$ & $\mathrm{Pa} \cdot \mathrm{s}$ & $\mathrm{ML}^{-1} \mathrm{~T}^{-1}$ \\
$C_{p}$ & Specific heat & $\mathrm{J} / \mathrm{kg}-\mathrm{K}$ & $\mathrm{L}^{2} \mathrm{~T}^{-2} \mathrm{~K}^{-1}$ \\
$u$ & Velocity & $\mathrm{m} / \mathrm{s}$ & $\mathrm{LT}^{-1}$ \\
\hline
\end{tabular}

The Buckingham $\Pi$-theorem for dimensional analysis [13] was used to produce the following expression for the heat-transfer coefficient as a function of the identified heat-transfer parameters (the associated terms of empirical correlation appear in Table 3-3.

$$
h=f\left(D, z, k_{b}, \rho_{b}, \mu_{b}, C_{p}, u\right)
$$


TABLE 3-3 $\Pi$-terms of empirical correlation.

\begin{tabular}{lcl}
\hline \multicolumn{1}{c}{$\Pi$-terms } & Dimensionless group & \multicolumn{1}{c}{ Name } \\
\hline$\Pi_{1}$ & $\frac{h D}{k_{b}}$ & Nusselt number \\
$\Pi_{2}$ & $\frac{D u \rho_{b}}{\mu_{b}}$ & Reynolds number \\
$\Pi_{3}$ & $\frac{C_{p} \mu_{b}}{k_{b}}$ & Prandtl number \\
$\Pi_{4}$ & $\frac{z}{D}$ & Non-dimensional axial \\
\hline
\end{tabular}

$\Pi_{1}=f\left(\Pi_{2}, \Pi_{3}, \Pi_{4}\right)$

$N u=4.36+C \cdot \operatorname{Re}_{b}^{n 1} \operatorname{Pr}_{b}^{n_{2}}\left(\frac{z}{D}\right)^{n_{3}}$

$\log (N u-4.36)=\log (C)+n_{1} \log \left(R e_{b}\right)+n_{2} \log \left(P r_{b}\right)+n_{3} \log \left(\frac{z}{D}\right)$

$\mathrm{Y}=\mathrm{A} \mathrm{X}$

$\mathrm{A}=\left[\begin{array}{cccc}1 & \log \left(R e_{i=1}\right) & \log \left(P r_{i=1}\right) & \log \left(\frac{z_{i=1}}{D}\right) \\ \vdots & \ddots & & \vdots \\ 1 & \log \left(R e_{i=N}\right) & \log \left(P r_{i=N}\right) & \log \left(\frac{z_{i=N}}{D}\right)\end{array}\right]$

$\mathrm{X}=\left[\begin{array}{c}\log (C) \\ n_{1} \\ n_{2} \\ n_{3}\end{array}\right]$

$\mathrm{Y}=\left[\begin{array}{c}\log \left(N u_{i=1}-4.36\right) \\ - \\ - \\ \log \left(N u_{i=N}-4.36\right)\end{array}\right]$

In Eq. 8, the output ' $\mathrm{Y}$ ' and matrix ' $A$ ' are known variables. The ' $\mathrm{X}$ ' is the unknown parameter, which needs to be predicted by the Matlab regression function. The regression model returns the unknown parameters for ' $\mathrm{X}$ ' (Eq. 10) upon being provided the known variable data. 


\subsection{MACHINE LEARNING MODEL}

The open source deep learning library PyTorch is used to develop the machine learning model, which is in the form of the Deep Feed-forward Neural Network (DFNN). The basic architecture of DFNN) is illustrated in Figure 3-2. In the network, the first layer is the input and the last layer is the output; in between are the hidden layers. The units in the hidden layers are also termed "neurons" and are represented by a nonlinear function of a certain form. The term "deep" indicates that the feed-forward network has multiple hidden layers, while the term "feed-forward" means that there are no feedback connections in the hidden layers.

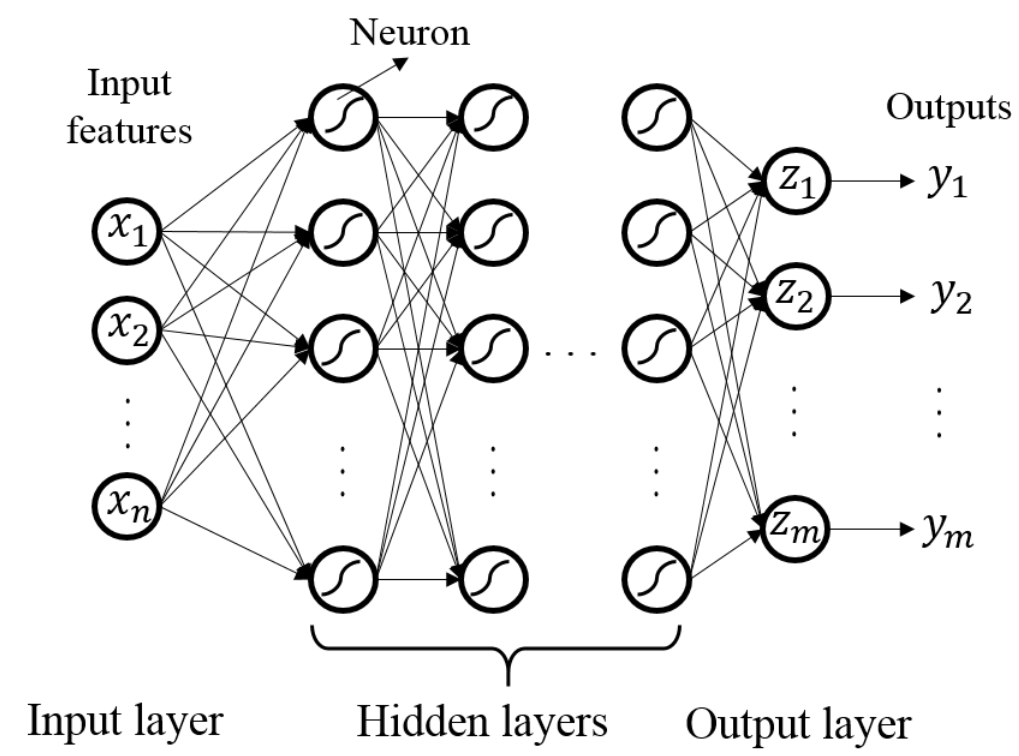

FIGURE 3-2 Architecture of a fully connected, deep feed-forward neural network.

Essentially, the DFNN can be regarded as a process where the input features go through a series of nonlinear transformations to predict the outputs. The behavior of the DFNN is controlled by learnable parameters, weights $\boldsymbol{W}$ and biases $\boldsymbol{b}$, followed by a nonlinear function called the activation function $g(x)$. In this sense, the neural network in Figure 3-2 can be interpreted as the following transformations:

$$
\begin{gathered}
\boldsymbol{h}_{1}=g\left(\boldsymbol{W}_{1}^{\boldsymbol{T}} \boldsymbol{x}+\boldsymbol{b}_{1}\right) \\
\boldsymbol{h}_{2}=g\left(\boldsymbol{W}_{2}^{T} \boldsymbol{h}_{1}+\boldsymbol{b}_{2}\right) \\
\vdots \\
\boldsymbol{y}=g\left(\boldsymbol{W}_{l+1}^{\boldsymbol{T}} \boldsymbol{h}_{l}+\boldsymbol{b}_{l+1}\right)
\end{gathered}
$$

In practice, there are many choices for use as the activation function, including sigmoid function, tanh function, and rectified linear units (ReLUs). The tanh function and the sigmoid function have been applied in many previous artificial neural network (ANN) applications with 
only a few hidden layers and have worked well for these applications. However, these two functions face the problem usually termed "saturated neurons" when dealing with deeper neural networks. When the absolute value of the input of a tanh/sigmoid function is large (for example, $x>10$ or $x<-10$ ), the function is "flat," so the change of input will result in a near-zero gradient. The "saturated neurons" create difficulties in obtaining the desired gradient flow that is vital for the training of networks. Such an issue is tolerable in ANNs with only a few hidden layers, but it becomes serious for deeper networks. In this work, an ReLU is used as the activation function, which can be expressed as follows:

$$
g(x)= \begin{cases}0, & x<0 \\ x, & x \geq 0\end{cases}
$$

With the input features, number of hidden layers, number of hidden units, weights $\boldsymbol{W}$ and biases $\boldsymbol{b}$, activation function, and outputs setup, the architecture of the DFNN is determined. The next step is to train the DFNN with enough data; generally speaking, the deeper the network, the more data required for training. For training a DFNN, a loss function $L(\hat{\boldsymbol{y}}, \boldsymbol{y})$ needs to be defined to measure the error between the DFNN's prediction $\widehat{\boldsymbol{y}}$ and the real data $\boldsymbol{y}$. For physical problems, the $L_{2}$ norm loss functions, also known as the mean square error (MSE), is the most widely used:

$$
L_{2} \text { norm: } L(\hat{\boldsymbol{y}}, \boldsymbol{y})=\|\widehat{\boldsymbol{y}}-\boldsymbol{y}\|_{2}=\frac{1}{m} \sum_{i=1}^{m}\left(\hat{y}_{i}-y_{i}\right)^{2}
$$

In this work, the MSE is used as the error function in the training of the network; its root, the root of mean square error (RMSE), is used to evaluate the performance of the model. Besides the RMSE, the coefficient of determination $\left(\mathrm{R}^{2} \mathrm{score}\right)$ is also used to evaluate the performance of the trained model:

$$
R^{2}=1-\frac{\sum_{i=1}^{m}\left(\hat{y}_{i}-\overline{\hat{y}}\right)^{2}}{\sum_{i=1}^{m}\left(\hat{y}_{i}-\bar{y}_{l}\right)^{2}}
$$

Once the loss function is defined, the error gradients with regard to the weights and biases of each layer can be computed through backpropagation based on the chain rule of calculus. In the practice of training a deep DFNN, there is one common issue: overfitting. If a DFNN is overfitted with the training data, the total loss function will be small, but it will fail to predict a dataset that is not included in the training. In this work, the dataset is divided into two parts: about $70 \%$ of the data is used for training, while the remaining $30 \%$ of the data is used for testing. Two approaches are applied in the training process to mitigate the overfitting issue.

The first approach is to include a regularization term $\boldsymbol{\Omega}(\boldsymbol{\theta})$ in the loss function $L$ :

$$
\tilde{J}(\boldsymbol{\theta} ; \hat{\boldsymbol{y}}, \boldsymbol{y})=J(\boldsymbol{\theta} ; \hat{\boldsymbol{y}}, \boldsymbol{y})+\lambda \boldsymbol{\Omega}(\boldsymbol{\theta}),
$$


where $\lambda$ is a positive hyperparameter that weights the relative contribution of the regularization term, where a large $\lambda$ indicates strong regularization. In most practices, the regularization term is only applied to weights; thus, $\boldsymbol{\Omega}(\boldsymbol{\theta})$ is equal to $\boldsymbol{\Omega}(\boldsymbol{w})$. The most widely used weights $\boldsymbol{\Omega}(\boldsymbol{w})$ are $L_{1}$ and $L_{2}$ regularization:

$$
\begin{gathered}
L_{1} \text { regularization: } \boldsymbol{\Omega}(\boldsymbol{w})=\|\boldsymbol{w}\|_{1}=\sum w_{i} \\
L_{2} \text { regularization: } \boldsymbol{\Omega}(\boldsymbol{w})=\frac{1}{2} \boldsymbol{w}^{T} \boldsymbol{w}
\end{gathered}
$$

In this work, the $L_{2}$ regularization is in the training process, and the value of $\lambda$ is tuned to minimize the RMSE of the testing dataset.

The second approach applied in this work is to introduce a dropout rate into the training process, such that dropout layers are inserted into the hidden layers. During training, the dropout layer will randomly zeroed some of the elements of the input with probability $p$ using samples from a Bernoulli distribution. Such an approach has proven to be an effective technique for regularization and preventing the overfitting issue.

In this work, the open-source deep learning library PyTorch is used, on the basis of which a six-layer DFNN is constructed, with a dropout layer inserted into the 2nd, 4th, and 6th hidden layers. Each layer consists of 200 neurons. The scripts of the network and the training are included in Appendices A and B, respectively.

\subsection{RESULTS}

In this study, few data points were filtered out from the full dataset for providing the condition of $\mathrm{z} / \mathrm{D}<4$ and $\mathrm{Re} \leq 400$. Only three physical features, that is, the Reynolds number Re, Prandtl number Pr, and dimensionless length scale $z / D$, were considered in this case study. This choice is consistent with the derivation of one of the empirical correlations (Eqs. 2 and 5).

\subsubsection{Matlab Regression Model}

In Figure 3-3, the uncertainty using the Matlab regression model is shown, which is up to $\pm 30 \%$ (Eq. 18). The $\mathrm{R}^{2}$ value for model fit was 0.875 . The $\mathrm{R}^{2}$ values were calculated based on Eq. 15. It should be noted that the correlation and numerical data are identical if $R^{2}=1$. On the other hand, the correlation does not represent the numerical data if $\mathrm{R}^{2}=0$. The wall-heat transfer correlation is shown in Eq. (19).

$$
\begin{aligned}
& \text { Erorr } \%=\sum_{i=1}^{n} \frac{\left(N u_{\text {exp }}^{2}-N u_{\text {model }}^{2}\right)}{N u_{\text {exp }}^{2}} \times 100 \\
& N u=4.36+4.95 \cdot R e_{b}^{-0.074} \operatorname{Pr}_{b}^{-3.2731}\left(\frac{z}{D}\right)^{-0.5393}
\end{aligned}
$$




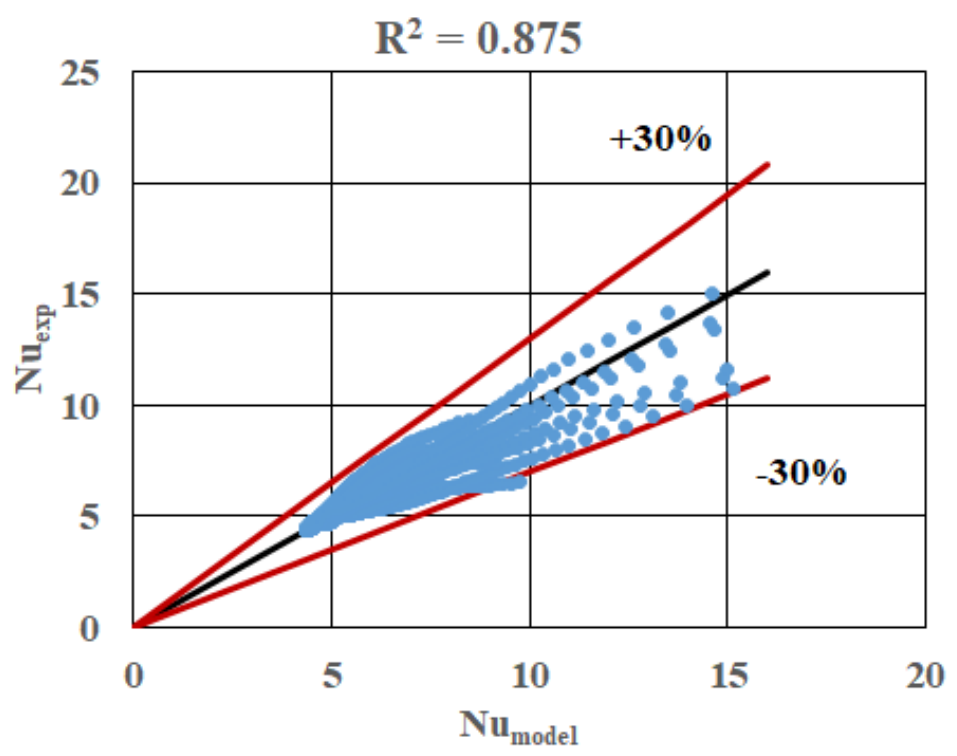

FIGURE 3-3 Comparison of Nusselt number for model and Nek5000 data.

\subsubsection{Machine Learning Model}

To establish the accuracy of the derived wall-heat transfer correlation, a comparison study is performed based on the deep learning technology. The DFNN is used as a "black-box" correlation that takes in the same physical features used in the empirical correlation to predict the Nusselt number. Such a network does not have a fixed functional form and thus is believed to have more flexibility to fully explore the feature space provided by the training dataset. The dataset is selected to filter out a few outlier points near the inlet of the flow. Only three features are used, such as local aspect ratio (z/D) and Reynolds and Prandtl numbers. Such a selection is consistent with the derivation of regression model.

Trained by 200 epochs, the RMSE and $\mathrm{R}^{2}$ score is plotted in Figure 3-4. It is evident that after being trained with 200 epochs, the RMSE of the training dataset and testing dataset reach the same level, while the $\mathrm{R}^{2}$ score increases to a high level. This finding demonstrates that the training process has been successful and the overfitting issue has been mitigated with the adopted regularization and dropout strategy. 

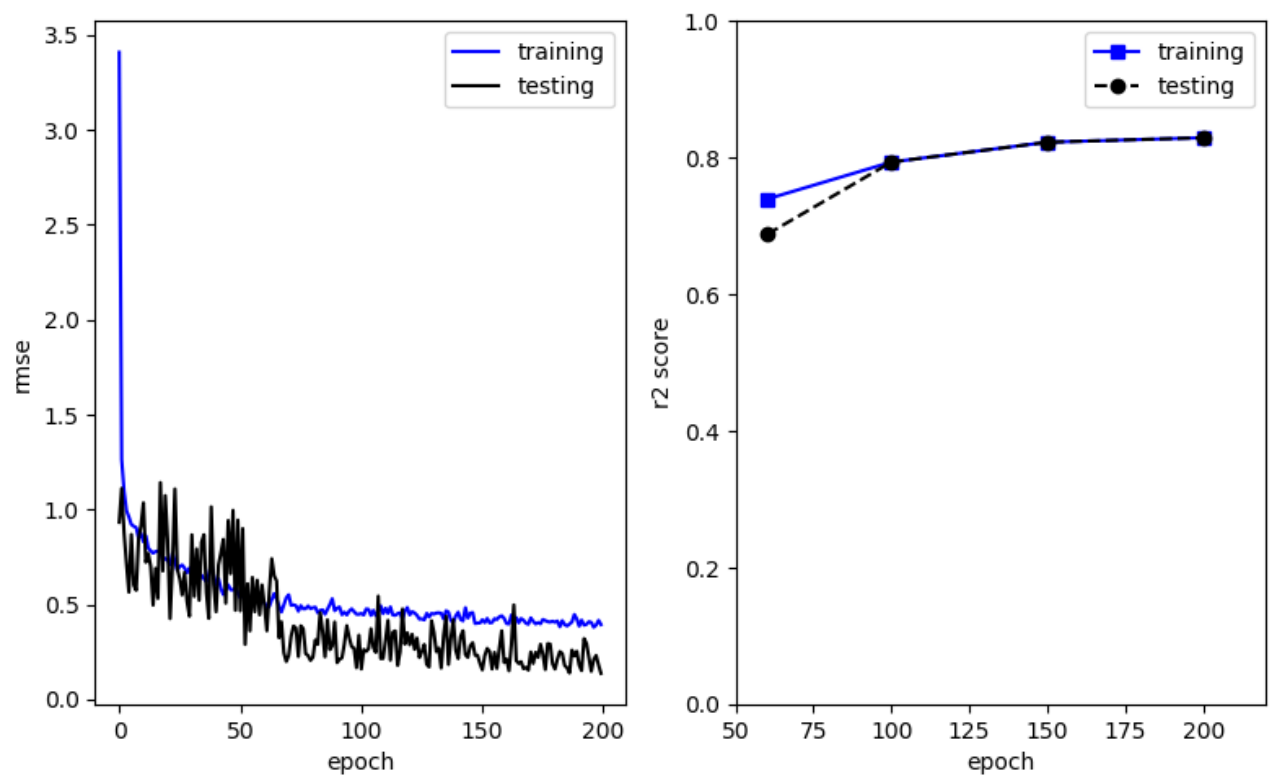

FIGURE 3-4 RMSE and $\mathbf{R}^{2}$ score for a data fit.

A direct comparison between the DFNN predictions and the original Nusselt number obtained from the Nek5000 simulations is depicted in Figure 3-5, and the relative error distribution is depicted in Figure 3-6. It is evident that the DFNN is still in very good agreement with the original Nek5000 data. On the other hand, a few points exhibit a larger discrepancy. The most probable reason that has led to such a discrepancy is that the network trained with only three features, neglecting some intrinsic pattern from the other features such as viscosity and the thermal conductivity's wall-to-bulk ratio.

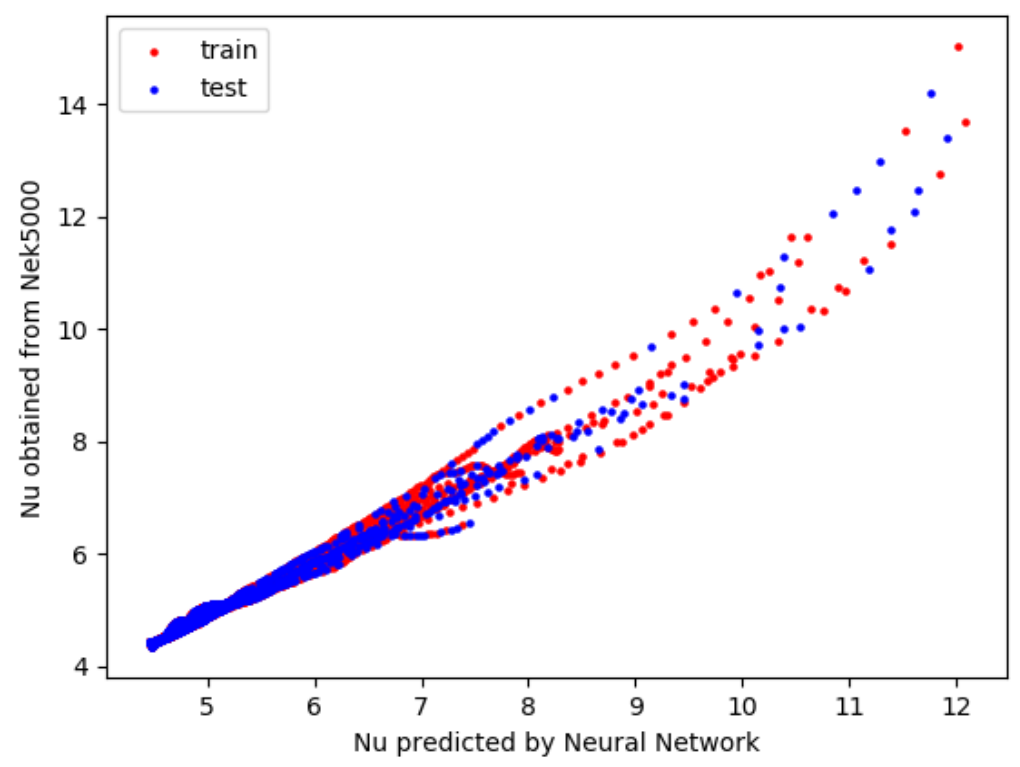

FIGURE 3-5 Direct comparison of DFNN predictions and original $\mathrm{Nu}$. 


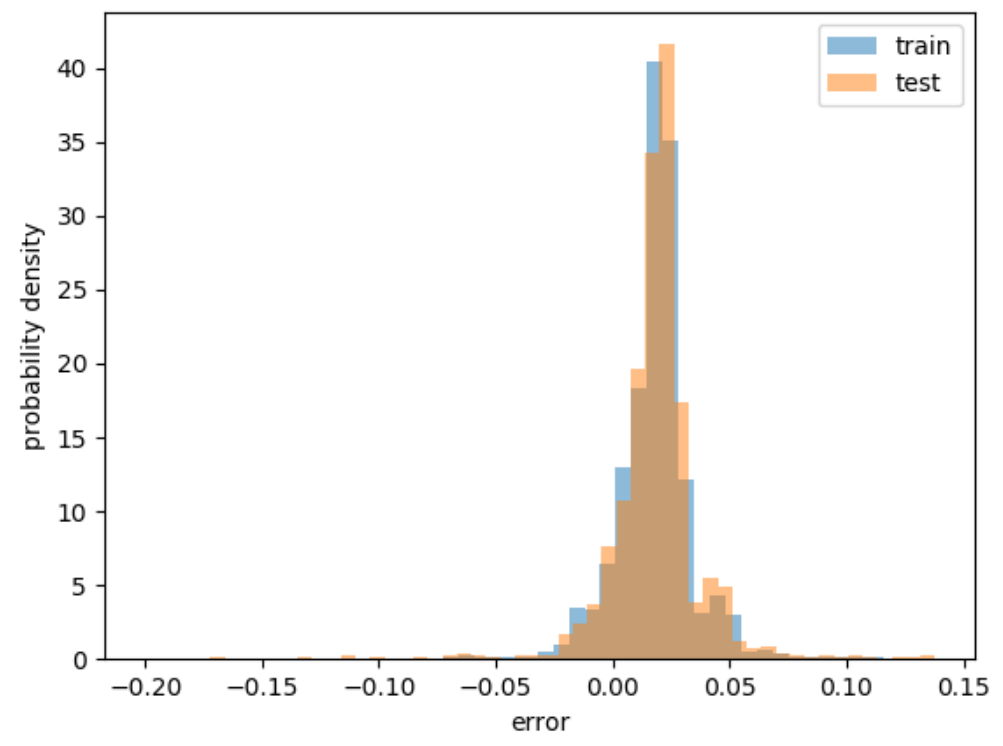

FIGURE 3-6 Histogram of relative error of DFNN predictions.

It should also be noted that because the trained DFNN is a "black-box" model, it has certain limitations when it is being applied to real engineering problems. The biggest limitation is that the trained DFNN is compatible with Python and can only be converted to a $\mathrm{C}++$ library through the Torchscript. If implementing the trained neural network into an existing tool, the weights and biases of each layer need to be manually coded into the existing code.

\subsection{CONCLUSIONS}

The Nek5000 CFD code was used to develop wall-heat transfer correlations for laminar flows. Several simulations were performed for flow in cylindrical geometries with various Reynolds numbers and wall-heat fluxes. Significant deviations were found when comparing the predicted heat-transfer coefficient and Sieder-Tate correlation.

The wall-heat transfer correlation was developed using the Matlab regression model. The developed correlation predicts the Nek5000 data within an error rate of $\pm 30 \%$. The $\mathrm{R}^{2}$ value for model fit was 0.875 , which ensures that $87.5 \%$ of the model data lies on the Nek5000 data. Furthermore, advanced numerical approaches such as machine learning tools were used to train and test the Nek5000 data. Good model fit was observed with machine learning tool. 


\section{FUTURE WORK}

Future work will continue to assess and apply the advanced modeling capabilities being developed under DOE-NE's Modeling and Simulation program to address key problems identified by the DOE-NE's Advanced Reactor Technology program or industry and that are important to the design and safety performance of HTGRs.

To improve the primary loop simulation of the MHTGR during transient conditions, we could include the heterogeneous modeling of heat structures within the fuel assembly for the graphite, cladding, and fuel to avoid the homogeneous assumption for fuel assemblies used in the current work. Furthermore, RCCS modeling can be improved by explicitly simulating the natural circulation of air flow. The developed heat transfer correlations using Nek5000 for laminar flow can be implemented in SAM $[8,14]$ and tested with MHTGR primary loop simulations using newly developed correlations.

The bypass flows in a prismatic GCR are of concern because they reduce the desired flow rates in the coolant channels and, thereby, can increase outlet gas temperatures and maximum fuel temperatures. In the existing literature, bypass flows of $10 \%$ to $30 \%$ of the total flow rate have been estimated [1]. Consequently, it is important to account for bypass flows in analyses of reactor thermal gas dynamics. Hence, the bypass flow effects in HTGRs should be addressed in future work. Other efforts would include continued engagements with industry on advanced modeling and simulation needs of HTGRs and performance of early demonstrations of interesting problems. Multi-scale modeling and simulation will also be pursued for thermal-hydraulic and safety performance analysis of advanced reactors by using and integrating engineering-scale simulation tools like Pronghorn and 1D system analysis codes like SAM, along with 3D tools like Nek5000. 


\section{REFERENCES}

1. Schultz, R., et al., "Identification and Characterization of Thermal Fluid Phenomena Associated with Selected Operating/Accident Scenarios in Modular High Temperature GasCooled Reactors,” Idaho National Laboratory, INL/EXT-17-43218 (2017).

2. Vegendla, P., et al., "Modeling and Simulations of Thermal-Fluid Phenomena Related to Pressurized Conduction Cooldown in High Temperature Gas Reactor,” Argonne National Laboratory, ANL-18/33 (2018).

3. Lee, J.I., et al., "Deteriorated Turbulent Heat Transfer of Gas Up-flow in a Circular Tube: Experimental Data,” International Journal of Heat and Mass Transfer, 51(1-2): 3259-3266 (2008).

4. Vegendla, P., et al., "Multi-Scale Modeling of Thermal-Fluid Phenomena Related to Loss of Forced Circulation Transient in HTGRs,” Argonne National Laboratory, ANL-19/35 (2019).

5. Vegendla, P., et al., "Deteriorated Turbulent Heat Transfer in Wall-Heated Cylindrical Tube," Journal of Verification, Validation and Uncertainty Quantification, 4 (2019).

6. Alwafi, A., et al., "Time-Resolved Particle Image Velocimetry Measurements of a Single Impinging Jet in the Upper Plenum of a Scaled Facility of High Temperature Gas-Cooled Reactors,” International Journal of Heat and Flow, 76: 113-129 (2019).

7. Vegendla, P., et al., "CFD Model Validation for a Benchmark Data of Texas A\&M 1/16" Scaled VHTR Upper Plenum,” ASME Verification and Validation Symposium, Baltimore, Maryland, May 2020.

8. Hu, R., "SAM Theory Manual," Argonne National Laboratory, ANL/NE-17/4, March 2017.

9. Novak, A.J., et al., Pronghorn Theory Manual, INL/EXT-18-44453, Idaho National Laboratory, 2018.

10. Fischer, P., et al., "Petascale Algorithms for Reactor Hydrodynamics,” J. Phys. Conf. Series (2008).

11. Lee, J.I., et al., "Deteriorated Turbulent Heat Transfer of Gas Up-Flow in a Circular Tube: Heat Transfer Correlations,” International Journal of Heat and Mass Transfer, 51(1-2): 53185326 (2008).

12. Sieder, E.N., et al., "Heat Transfer and Pressure Drop of Liquids in Tubes,” Industrial Engineering Chemistry, 28: 1429-1435 (1936).

13. Munson, B., et al., Fundamentals of Fluid Mechanics (fifth ed.), New York, NY: J. Wiley \& Sons (2005). 
14. Hu, R., L. Zou, and G. Hu, "SAM User’s Guide,” Argonne National Laboratory, ANL/NSE19/18, Argonne, IL, August 2019. 


\section{APPENDIX A: NETWORK SCRIPTS}

\#! Trained and Tested Models

\#!/usr/bin/env python3

\# -*_ coding: utf-8 -*_

import numpy as np

import pandas as pd

import torch

import random

import pickle

from FNN import FF_Net

from torch.optim.lr_scheduler import ReduceLROnPlateau

import torch.utils.data as Data

import torch.optim as optim

import torch.nn.functional as $\mathrm{F}$

data_partial = pd.read_excel('data_not included zbyD_lt_4 and Re_lt_400.xlsx')

data_partial = data_partial.astype(float)

random.seed(1)

partial_index = range(len(data_partial))

train_index = sorted(random.sample(range(len(data_partial)),int $(0.7 * \operatorname{len}($ data_partial $)))$ )

test_index = sorted(list(set(partial_index) - set(train_index))

input_train = data_partial[['Re','Pr','x/Dia']].iloc[train_index $]$

input_train = torch.from_numpy(input_train.values)

input_train = input_train.type(torch.FloatTensor)

output_train = data_partial['Nu_exp'].iloc[train_index]

output_train = torch.from_numpy(output_train.values)

output_train = output_train.type(torch.FloatTensor).unsqueeze(-1)

input_test = data_partial[['Re','Pr','x/Dia']].iloc[test_index $]$

input_test $=$ torch.from_numpy(input_test.values)

input_test = input_test.type(torch.FloatTensor)

output_test $=$ data_partial['Nu_exp'].iloc[test_index]

output_test = torch.from_numpy(output_test.values)

output_test = output_test.type(torch.FloatTensor).unsqueeze(-1)

\# data_full = pd.read_excel('total_data.xlsx')

\# data_full = data_full.astype(float) 


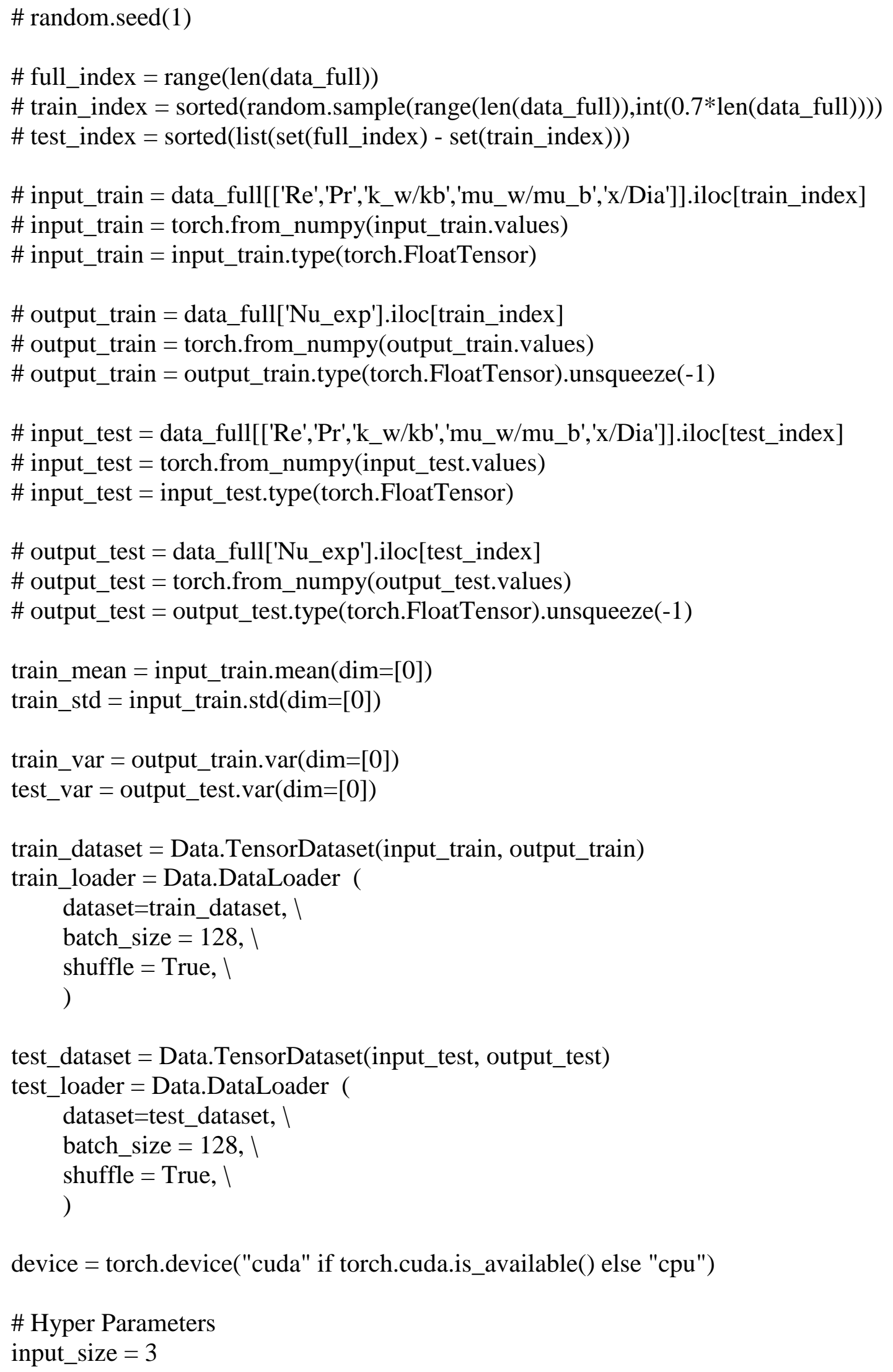




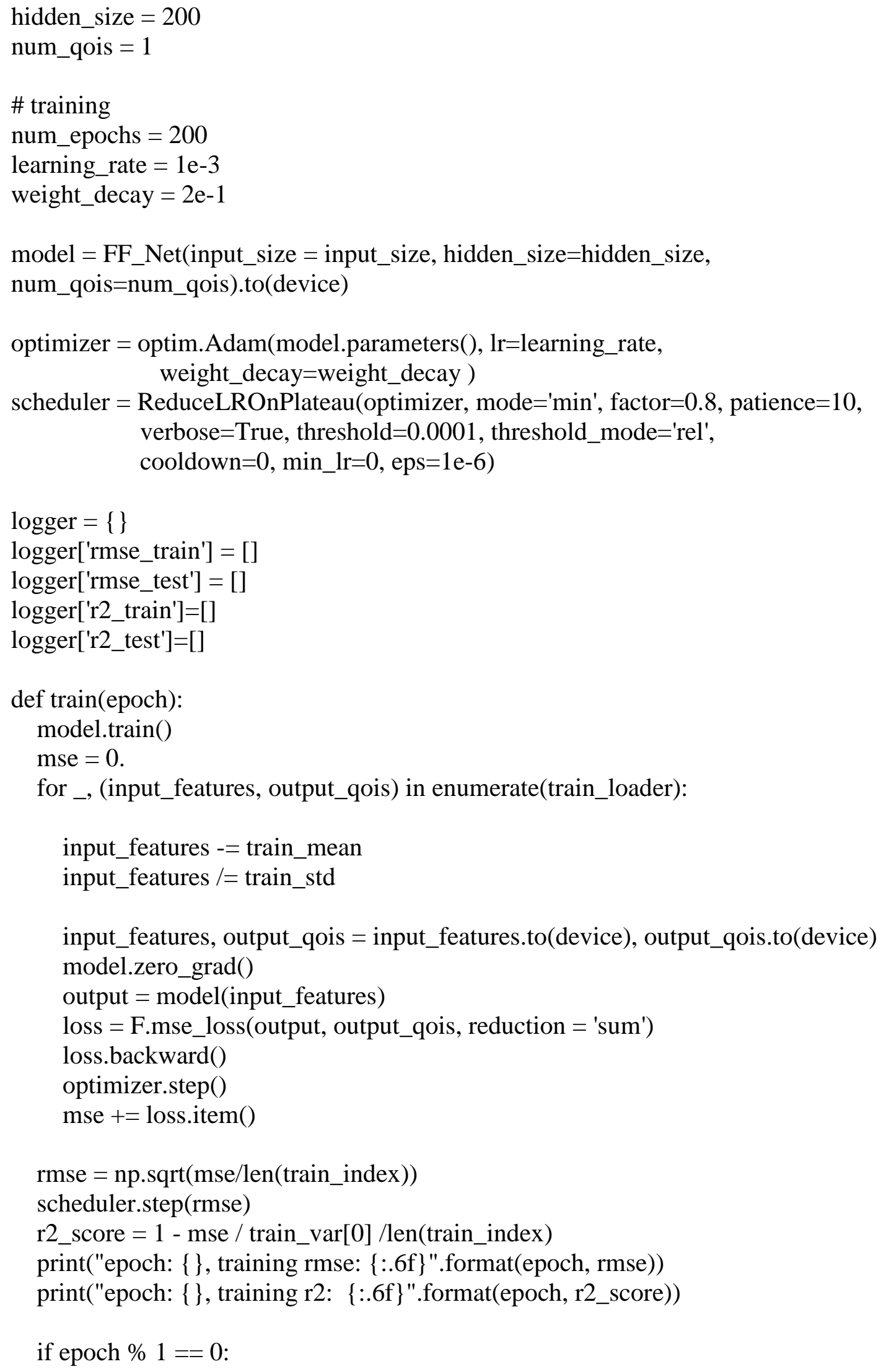


test(epoch)

\#! END Trained model 


\section{APPENDIX B: TRAINING OF THE NEURAL NETWORK}

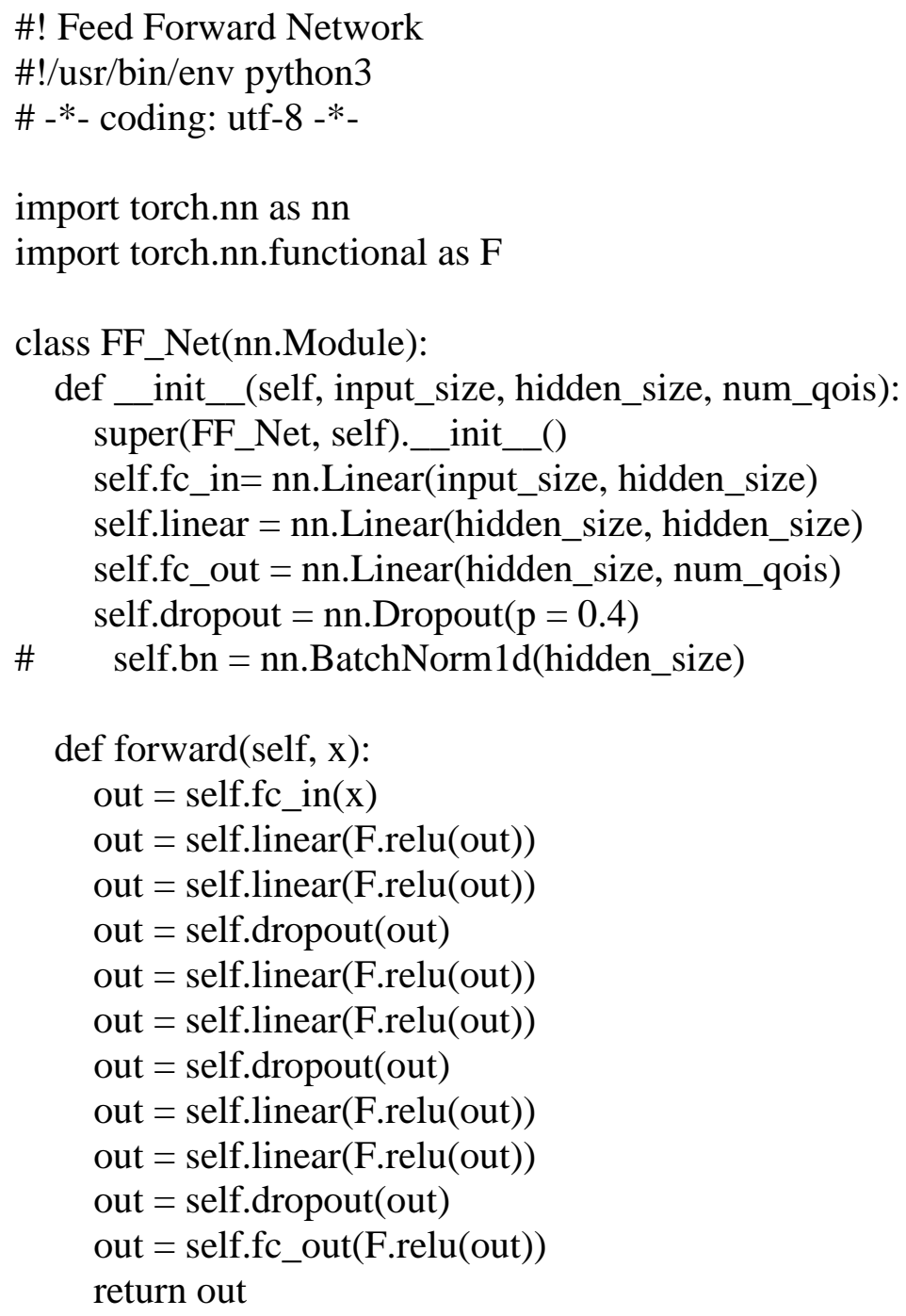


This page is intentionally left blank. 


\section{Argonne}

Nuclear Science and Engineering Division

Argonne National Laboratory

9700 South Cass Avenue, Bldg. 208

Argonne, IL 60439

www.anl.gov

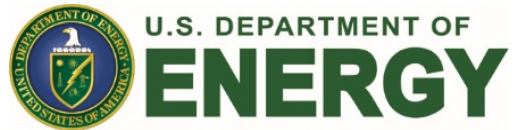

Argonne National Laboratory is a U.S. Department of Energy

laboratory managed by UChicago Argonne, LLC 